\title{
Synchronization of Fast (30-40 Hz) Spontaneous Cortical Rhythms during Brain Activation
}

\author{
M. Steriade, F. Amzica, and D. Contreras \\ Laboratoire de Neurophysiologie, Faculté de Médecine, Université Laval, Quebec, Canada G1K 7P4
}

We investigated the synchronization of fast spontaneous oscillations (mainly $30-40 \mathrm{~Hz}$ ) in anesthetized and behaving cats by means of simultaneous extra- and intracellular recordings from multiple neocortical areas. Fast Fourier transforms, auto- and cross-correlations, and spike- or wave-triggered averages were used to determine the frequency and temporal coherence of fast oscillations that outlasted the stimulation of ascending activating systems or that occurred naturally during behavioral states of waking and rapid eye movement (REM) sleep but also appeared during the depolarizing phases of slow sleep oscillations. In $90 \%$ of microelectrode tracks, the fast oscillations did not show field reversal at any depth of the cortex and were not observable in the underlying white matter. The negative field potentials of the fast oscillations were associated at all depths with neuronal firing. This field potential property of fast oscillations was in sharp contrast to the reversal of slow sleep oscillation or evoked potentials at depths of $0.25-0.5 \mathrm{~mm}$. The coherence of fast spontaneous rhythms was spatially limited, being confined within a cortical column and among closely located neocortical sites, in contrast to the long-range synchronization of slow sleep rhythms. Depolarizing current pulses elicited spike-bursts $(200-400 \mathrm{~Hz})$ recurring at a frequency of $30-40 \mathrm{~Hz}$. Our experiments demonstrate that the conventional notion of a totally desynchronized cortical activity upon arousal should be revised as fast rhythms are enhanced and synchronized within intracortical networks during brain activation. Spontaneously occurring, subthreshold membrane potential depolarizing oscillations may bias cortical and thalamic neurons to respond synchronously, at fast frequencies, to relevant stimuli in the wake state or to internally generated drives in REM sleep.

Key words: fast rhythms; depolarization; cortex; sleep; arousal; synchronization
This study challenges the conventional notion according to which the electrical activity of the brain is completely desynchronized upon arousal. Instead, we demonstrate that although low-frequency sleep oscillations are suppressed, fast activities (mainly $30-40 \mathrm{~Hz}$ ) are enhanced and synchronized within cortical networks during brainstem-elicited activated epochs as well as during natural slates of waking and rapid eye movement (REM) sleep.

The effect of brainstem reticular stimulation on electroencephalographic (EEG) activity has initially been described in acute experiments under the term "activation" (Moruzzi and Magoun, 1949), despite no evidence at that time that cortical and thalamic responsiveness is potentiated upon arousal. A decade later it was shown that stimulation of the brainstem reticular core increases the amplitudes of evoked potentials in sensory neocortical areas (Bremer and Stoupel, 1959; Dumont and Dell, 1960) and visual thalamus, without being accompanied by similar changes in responses recorded simultaneously from the optic tract (Steriade and Demetrescu, 1960). In the pioneering article by Moruzzi and Magoun (1949), the activation of EEG mainly consisted of obliteration of slow waves. When present, fast activity had a low voltage; during deep anesthesia, "fast activity was not elicited" (p. 456). With the notable exception of an incidental

Received May 25, 1995; revised Aug. 31, 1995; accepted Sept. 13, 1995.

This work was supported by the Medical Research Council of Canada (Grant MT-3689). F.A. and D.C. are graduate students, partially supported by the Fonds de Recherche en Santé du Québec and the Savoy Foundation. We thank R. R. Llinás and D. Paré for helpful remarks on an earlier version of this manuscript, I. Timofeev and A. Nuñez for collaboration in two experiments, and G. Oakson, P. Giguère, and D. Drolet for technical assistance.

Correspondence should be addressed to $M$. Steriade at the above address. Copyright (c) 1995 Society for Neuroscience $0270-6474 / 95 / 160392-26 \$ 05.00 / 0$ observation (see Fig. 5 in Bremer et al., 1960), most subsequent studies similarly reported a flattened electrical activity of the cortex during and shortly after brainstem core stimulation.

Today the interest in fast rhythms is attributable to numerous studies describing oscillations at $20-60 \mathrm{~Hz}$, as evoked under various behavioral conditions of increased alertness (Lopes da Silva, 1970; Bouyer et al., 1981; Freeman and Van Dijk, 1987; Murthy and Fetz, 1992) or appearing as responses to optimal sensory stimuli in animals and humans (Eckhorn ct al., 1988; Gray et al., 1989; Pantev et al., 1991; Jagadeesh et al., 1992; Llinás and Ribary, 1993; Desmedt and Tomberg, 1994; Gray, 1994). Although some consider the photically evoked fast rhythms to be generated exclusively in cortical networks, similar oscillations are induced by visual stimuli in the thalamic lateral geniculate nucleus and retina (Steriade, 1968; Ghose and Freeman, 1992). Many authors designate such fast oscillations under the term of $40 \mathrm{~Hz}$ oscillations, which would indicate a fixed or preferred frequency. The attraction for this magic frequency ignores the fact that rhythms implicated in attentive processes or produced by optimal sensory signals extend over a rather broad spectrum, from as low as $14 \mathrm{~Hz}$ to as high as $80 \mathrm{~Hz}$ (Frégnac et al., 1994).

As for the fast spontaneous rhythms, cortical oscillations between 20 and $40 \mathrm{~Hz}$ outlast the stimulation of generalized ascending activating systems (Steriade et al., 1991b, 1993a). Fast rhythms appear in the cortex during brain-active states of waking and REM sleep in humans and in animals at the magnetoencephalographic (MEG) and EEG levels (Llinás and Paré, 1991; Llinás and Ribary, 1993; Franken et al., 1994; Llinás et al., 1994; Uchida et al., 1994). At the cellular level, rhythmic (30-40 Hz) spikebursts with unusually high intraburst frequencies $(900-1000 \mathrm{~Hz}$ ) 
occur during waking and REM sleep in rostral intralaminar thalamocortical neurons with peculiar electrophysiological properties (Steriade et al., 1993b).

The present study deals with the following: (1) the intracortical depth profile of spontaneously occurring fast oscillations and the relations between field potentials and extracellular/intracellular activities within the frequency range of $20-40 \mathrm{~Hz}$; (2) the spatial extent of coherency among cortical neuronal pools displaying fast oscillations; (3) the similarity between fast activities outlasting brainstem reticular stimulation and those occurring during natural brain-activated states of wakefulness and REM sleep; and (4) the fast spontaneous oscillations that also appear in neocortical cells during the depolarizing phase of the slow oscillation characterizing the state of resting sleep.

\section{MATERIALS AND METHODS}

The experiments were performed on acutely prepared cats and on chronically implanted cats. For acutc cxpcriments, the animals werc ancsthetized with ketamine and xylazine (10-15 mg/kg; $2-3 \mathrm{mg} / \mathrm{kg}$, i.m.). The tissues to be incised and the pressure points were infiltrated with a local anesthetic. The animals were paralyzed with gallamine triethiodide and artificially ventilated with control of end-tidal $\mathrm{CO}_{2}$ at $3.5-3.8 \%$. Body temperature was maintained at $37-39^{\circ}$, and heartbeat was monitored. The EEG was continuously recorded to maintain a constant sleep-like state, and additional doses of ketamine and xylazine were administered at the slightest EEG change that indicated a tendency toward an activated pattern. Chronic implantation of electrodes for cardinal signs distinguishing natural wake and sleep states [EEG, electromyogram (EMG), and electro-oculogram (EOG)] were performed under ketamine $(15 \mathrm{mg} / \mathrm{kg}$, i.m.) followed by barbiturate anesthesia ( $25 \mathrm{mg} / \mathrm{kg}$, i.p.). Atropine sulfate $(0.05 \mathrm{mg} / \mathrm{kg}$, i.m. $)$ was injected to prevent secretions. Buprenorphine $(0.03 \mathrm{mg} / \mathrm{kg}, \mathrm{i} . \mathrm{m}$.) was given every $12 \mathrm{hr}$ for $24 \mathrm{hr}$ to prevent pain after surgery. An antibiotic (bicillin) was injected intramuscularly for $3 \mathrm{~d}$ postoperatively. Recordings began 7-10 d after surgery and continued for 2-3 weeks. Animals were not deprived of sleep. The method used to keep the head rigid without pain or pressure during the recording sessions was similar to that described previously (Steriade and Glenn, 1982). During recording, the animal could move its limbs and often made postural adjustments.

Recording and stimulation. The global electrical activity of the cortex was recorded with the following: (1) coaxial macroelectrodes, with the ring placed on the cortical surface and the tip inserted at a depth of 0.8-1 $\mathrm{mm}$ [the electrodes were inserted perpendicularly to the surface and produced either bipolar potentials (tip-ring) or monopolar potentials (one of the leads vs a reference placed on muscle)]; (2) circular arrays of eight macroelectrodes separated by $0.25 \mathrm{~mm}$ at different depths with the upper electrode at the cortical surface; and (3) a ring placed on the surface through which tungsten microwires or glass micropipettes were inserted to record field potentials and unit discharges at different depths.

Intracellular recordings of cortical neurons were performed with glass micropipettes filled with a $3 \mathrm{M}$ solution of potassium acetate (impedance $25-35 \mathrm{M} \Omega$ ). The stability of intracellular recordings was achieved by performing a bilateral pneumothorax, draining the cisterna magna, suspending the hip, and filling the hole made for the recordings with a solution of $4 \%$ agar. The micropipettes were placed $\sim 1 \mathrm{~mm}$ apart from the coaxial recording macroelectrodes, or they penetrated the cortex through the recording ring placed on the surface. For extracellular unit or multiunit discharges and field potentials, tungsten microwires or coarser glass micropipettes (resistances 1-5 M $\Omega$ ) were used. Microelectrodes were inserted perpendicular to the cortical surface, and the depth was read on the scale of the micromanipulator. As shown elsewhere (Steriade et al., 1993d; Contreras and Steriade, 1995), differences between micromanipulator readings and location of intracellularly stained neurons by means of Lucifer yellow or Neurobiotin were $<15 \%$. A high-impedance dual amplifier with active bridge circuitry was used to record and inject current into neurons. The signals were recorded on an eight-channel tape with a bandpass of $0-9 \mathrm{kHz}$, digitized at $20 \mathrm{kHz}$ for off-line computer analysis.

Spontaneous EEG activation occurred occasionally for short epochs $(5-10 \mathrm{sec})$. When longer periods $(10-20 \mathrm{sec})$ were required, the pedunculopontine tegmental (PPT) or laterodorsal tegmental (LDT) cholinergic nuclei, locus coeruleus, or deep cerebellar fastigial or interpositus nuclei were stimulated with trains of pulses $(0.1-0.2 \mathrm{msec}, 0.05-0.8 \mathrm{~mA})$ delivered through bipolar electrodes.

Data analysis. The purpose of the analyses was to disclose time relations among different oscillating foci, their frequency spectrum, and some dynamic aspects characterizing these relations. Time relations were studied with cross-correlation functions (Bendat and Piersol, 1980) that contain a central peak, the amplitude of which, between -1 and +1 , provides information about the degree of resemblance and the phase sign of the two waves, whereas the abscissa yields to the time lag separating the two waves. The cross-correlation function also contains information about the common spectral features of the underlying waves, but this is better unveiled by fast Fourier transforms (FFTs). Generally, the principal frequencies were disclosed by applying FFTs directly to the raw data. This procedure is sensitive to the amplitude of the waves (ampler waves generate higher power spectra) but has the disadvantage of considering each wavelet as part of a sinusoid; thus, the power spectrum is contaminated with spurious events (Barlow, 1993). To avoid this, we also produced the FFTs from autocorrelation functions, which show the same main peak frequencies as those of the raw data but discard the inconsistent oscillations. In addition, by deriving from an autoscaled function, direct spectral comparisons can be made among waves with independently evolving amplitudes. To selectively uncover activities in a particular frequency band, the recordings were filtered digitally.

The procedures for studying dynamic changes in the relations between waves were published elsewhere (Amzica and Steriade, 1995b). Briefly, the periods under observation were divided into successive windows, and correlational and spectral techniques were applied on the different channels belonging to a window. The resulting curves were assembled to form three-dimensional surfaces or their equivalent projections on the $x-y$ plane (topograms or contour maps).

The depth-profile analysis was performed via dual recordings, with one of the channels from the cortical surface (ring) and the other from a microelectrode that was lowered through the ring at successive depths in steps of $50-100 \mu \mathrm{m}$. At each depth, a period of at least 2 min was recorded. The two channels were digitally filtered for different frequency bands $(0-1,1-4,7-14$, and $15-80 \mathrm{~Hz})$, and the resulting couple of waves in each band were cross-correlated. The depth-profile analysis for a given band consists of two plots: the first displays the cross-correlation peaks versus the corresponding depths, and the second shows the evolution of time lags derived from the same cross-correlations as a function of the cortical depth of the microelectrode.

The influence of movements during recording sessions in behaving animals was determined by performing cross-correlations between active EEG leads and EMG or EOG. In none of the circumstances presented in this article did these correlations indicate a contribution to the EEG activity $>5 \%$.

After the experiments, animals were given a lethal dose of pentobarbital and perfused transcardially with physiological saline followed by $10 \%$ formaldehyde. The brain was removed and stored in formalin with $30 \%$ sucrose. The locations of microelectrode tracks and stimulating electrodes were verified on coronal or sagittal sections stained with thionine.

\section{RESULTS}

The data are presented as follows. First we compare the depth profile of fast oscillations, elicited in acute experiments by stimulation of mesopontine cholinergic nuclei, with the depth profile of the slow oscillation during sleep patterns. Next, inasmuch as the results show that fast oscillations are periodically suppressed during the long-lasting depth-positive EEG waves (reflecting cellular hyperpolarizations), we present intracellular data indicating the dependency of fast oscillations on the spontaneously occurring or current-elicited depolarization of neurons. Then, we analyze the spatial extent of the horizontal coherence of fast oscillations recorded from various neocortical foci. Finally, we present data from wake and sleep states in behaving animals to validate, under more natural conditions, the results obtained in acutely prepared animals under ketamine and xylazine anesthesia.

\section{Database}

The results are based on recordings of field potentials, extracellular unit discharges, and intracellular activities from various 


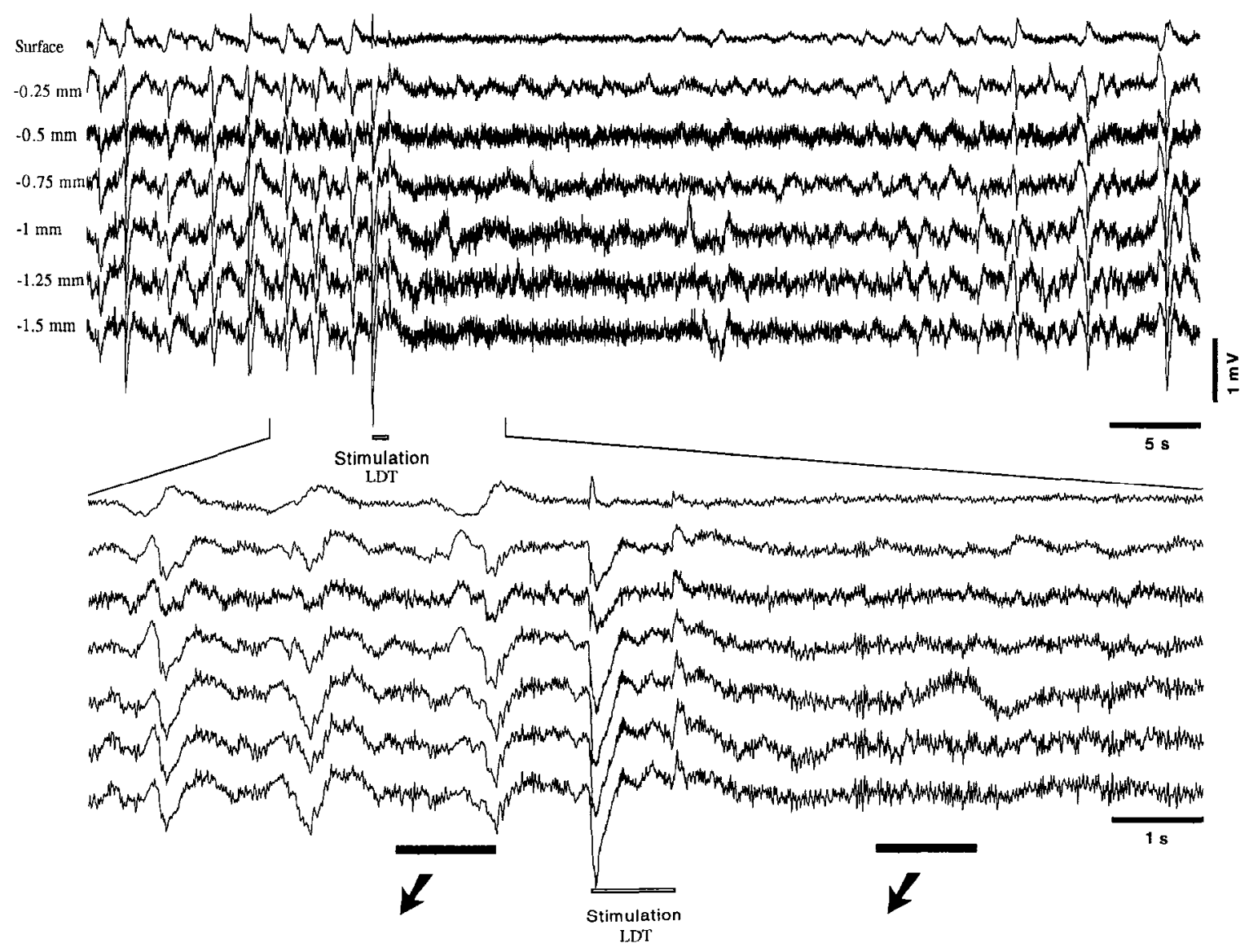

CROSS

\section{CROSS}
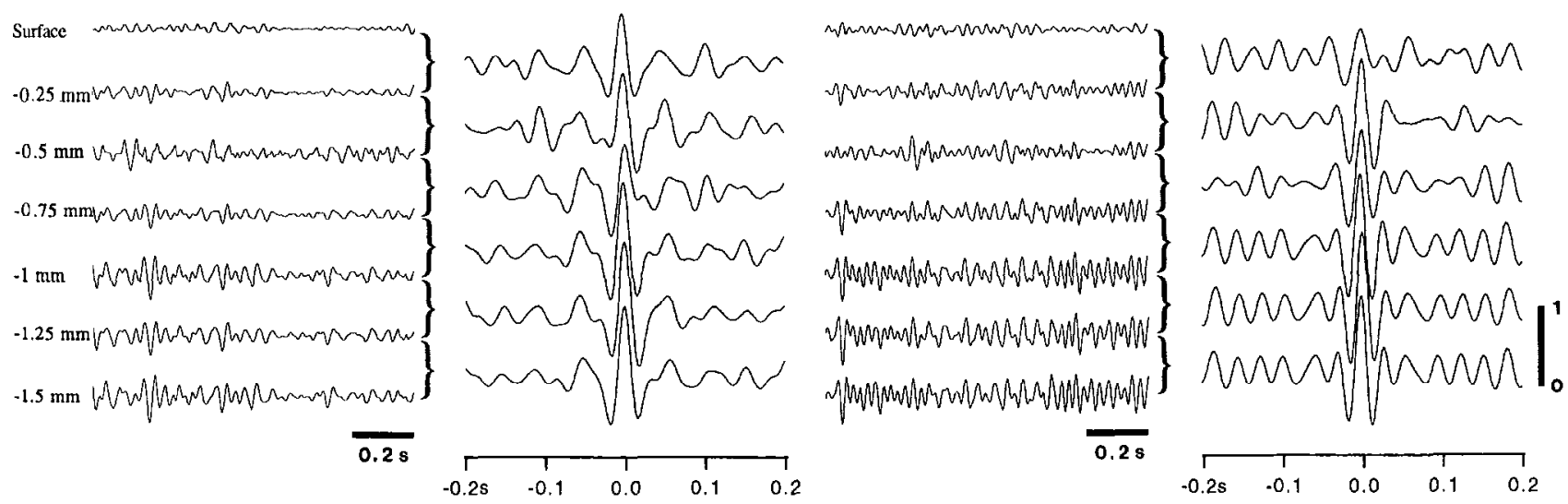

Figure I. Depth cortical profile of slow $(0.5 \mathrm{~Hz})$ cortical oscillation and fast $(\sim 35 \mathrm{~Hz})$ rhythms after stimulation of mesopontine cholinergic LD' nucleus. Ketamine and xylazine anesthesia. Simultaneous recording at the cortical surface and at different depths, from 0.25 to $1.5 \mathrm{~mm}$, through seven-electrode array (separated by $0.25 \mathrm{~mm}$ ) inserted in anterior suprasylvian area 5. The top panel shows a period of slow oscillation disrupted by LDT stimulation (pulse-train, $300 \mathrm{~Hz}, 0.9 \mathrm{sec}$ ). The LDT-elicited activation lasted for $\sim 30 \mathrm{sec}$; the slow oscillation resumed thereafter. The period before and after LDT stimulation (between lines) is extended below, showing the last three cycles of the slow oscillation and the activated epoch. Periods indicated by horizontal hars are further expanded below (arrows) and digitally filtered between 15 and $80 \mathrm{~Hz}$. The cross-correlations (CROSS) depicted at the right of each perind (before and after LDT stimulation) were computed over periods of $15 \mathrm{sec}$. Note: reversal of the components building up the slow oscillation at a depth of $0.25 \mathrm{~mm}$; fast activities are observed during both pre- and post-LDT periods, but they are suppressed during the prolonged depth-positive component of the slow oscillation (see especially the last oscillatory cycle before LDT stimulation), whereas after LDT stimulation they have a greater amplitude; at all depths, cross-correlations between activities from different depths seem to be in-phase. In this and in the following figures, polarity of EEG waves and extracellular activities is the same as for intracellular recordings (positivity up).

areas, as defined by cytoarchitectonic studies of cat cerebral cortex (Gurewitsch and Chatschaturian, 1928; Hassler and Muhs-Clement, 1964). In acutely prepared animals, the depth profile of slow and fast oscillations was investigated by means of simultaneous recordings at the surface and at different depths in 53 tracks within areas 6 and 4 of pericruciate gyri, anterior and posterior suprasylvian areas 5,7 , and 21 , and area 18 of the marginal gyrus. In addition, 23 other descents were made with the ring placed at the surface and with micropipettes or tungsten microwires penetrating through the ring, in successive steps of 50-100 $\mu \mathrm{m}$. Extra- 
cellular unit discharges were recorded simultaneously with local field potentials $(n=88)$. The fast oscillations of intracellularly recorded cortical neurons $(n=104)$, elicited by depolarizing pulses or appearing spontaneously during short activated epochs, were investigated in pericruciate areas 6 and 4, primary somatosensory (SI) cortex, and suprasylvian areas 5 and 7. The intracellular recordings that were used for analyses had overshooting action potentials and stable membrane potentials $\left(V_{\mathrm{m}}\right)$ more negative than $-60 \mathrm{mV}$ (without current) for at least $15 \mathrm{~min}$. Extracellular recordings of field potentials and unit discharges ( $n$ $=155$ ) were performed in chronically implanted, behaving animals during states of waking, resting sleep, and REM sleep, distinguished by conventional electrographic criteria (Steriade and McCarley, 1990). Thirty-nine complete wake-sleep cycles could be investigated; in addition, we analyzed 60 transitions from resting sleep to either waking or REM sleep. In chronic experiments, recordings were performed from areas $4,3,1,5$, and 7 .

\section{Depth profile of fast oscillation triggered by stimulation of ascending activating systems}

It was recently reported that fast $(20-40 \mathrm{~Hz})$ spontaneous neocortical rhythms can be induced by stimulating mesopontine PPT/ LDT cholinergic nuclei (Steriade et al., 1991a,b; 1993a), basal forebrain (Metherate et al., 1992), and cerebellar fastigial nuclei projecting over widespread cortical areas through a synaptic link in the ventromedial thalamic nucleus (Steriade, 1995).

Figure 1 depicts the effect of mesopontine LDT stimulation on the slow cortical oscillation $(0.5 \mathrm{~Hz})$. The slow oscillation of cortical neurons consists of prolonged depolarizing and hyperpolarizing components that recur periodically at $<1 \mathrm{~Hz}$ (Steriade et al., 1993d,e). The prolonged hyperpolarizations of neurons are reflected in the EEG as depth-positive waves, whereas the subsequent depolarizations are temporally related to sharp depthnegative EEG components followed by spindle waves and faster frequencies (Contreras and Steriade, 1995). In Figure 1, the LDT-induced blockade of the slow oscillation lasted for $\sim 30 \mathrm{sec}$, and during this activated epoch the fast EEG rhythms $(\sim 35 \mathrm{~Hz})$ displayed an increased amplitude.

The first step in the study of synchronization processes underlying the fast cortical activity was the analysis of its depth profile, compared with that of the slow oscillation. Although the main components of the slow oscillation recorded at the cortical surface were reversed at a depth of $0.25-0.5 \mathrm{~mm}$, the fast oscillation did not reverse from the surface to a depth of $1.5 \mathrm{~mm}$. This was clearly seen by visual inspection of activities from the surface and at various depths, filtered between 15 and $80 \mathrm{~Hz}$ (see expanded details of filtered fast activities in Fig. 1) and was further confirmed by cross-correlations between successive channels. The correlations, computed from periods before and after LDT stimulation (Fig. 1), show central peaks appearing in-phase and more regular and higher amplitude fast waves after LDT stimulation, visible in the surface-to $-0.25 \mathrm{~mm}$ correlation but especially among deep layers (1-1.5 mm depth).

To compare the depth profiles of the slow and fast oscillations, field potentials from sleep-like epochs and from LDT-activated periods were digitally filtered between both $0-4 \mathrm{~Hz}$ and $15-80 \mathrm{~Hz}$ (Fig. 2A). The main features revealed by these analyses are as follows. (1) The blockade of the slow oscillation $(0.7 \mathrm{~Hz})$ was associated with incrcased amplitudes of fast waves $(35-40 \mathrm{~Hz})$, exceeding those seen before LDT stimulation; we thus emphasize that the fast waves are present not only after the arousing stimulation but also before, during sleep-like patterns. (2) Fast rhythms were sustained during the activated epoch, but they were interrupted during the depth-positive component of the slow sleep oscillation (asterisks in the $1.25 \mathrm{~mm}$ and $1.5 \mathrm{~mm}$ traces of Fig. $2 A$, top). (3) Although the slow oscillation reversed polarity at cortical depths between 0.25 and $0.5 \mathrm{~mm}$, the fast rhythms were found in phase throughout the cortex (see phase opposition of slow oscillation between electrodes $1-2$ and 2-3 and in-phase correlations of fast rhythms from the surface to deep layers in Fig. 2A).

In-phase, surface, and depth, self-sustained fast activities were also observed by using very brief stimuli consisting of two short delayed volleys to the mesopontine cholinergic nuclei. Such stimuli evoked an initially surface-positive field potential that reversed between 0.25 and $0.5 \mathrm{~mm}$, followed by fast waves $(35-40 \mathrm{~Hz})$ that displayed the same polarity from the surface to the deepest cortical layers (Fig. $3 A$ ). Cross-correlations between surface- and depth-recorded potentials showed synchronized, in-phase fast oscillation lasting for $600 \mathrm{msec}$ (Fig. 3B). Clear-cut in-phase correlations were obtained only after $\sim 300 \mathrm{msec}$ after the PPT volleys (second window), when the fast rhythms were fully organized after the development of the early brainstem-thalamocortical response resembling the ponto-geniculo-occipital (PGO) potential (see Discussion).

The puzzling finding that fast rhythms do not display depth reversal was first observed in suprasylvian (association) area 5 and thereafter in coronal (somatosensory) area $3 b$ and precruciate (motor) area 6. The results were similar to simultaneous recordings from the surface and from different depths (as in Figs. 1, 2A, and 3 ) and with tungsten microelectrodes inserted through a ring placed on the surface, entering the cortex in steps of 50-100 $\mu \mathrm{m}$ (Fig. $2 B$ ). These data raised the obvious question whether such an activity is volume-conducted from sources extrinsic to the explored area. It should be emphasized again that in contrast to the absence of reversal of fast rhythms, the slow spontaneous oscillation or conventional evoked potentials displayed clear reversal, suggesting a large sink in the cortical depth below $0.3-0.4 \mathrm{~mm}$ and a source in the superficial layers (Figs. 1-3).

The first argument against the possibility of volume-conducted fast oscillation was the significantly decreased amplitudes of fast waves when the electrodes recorded the electrical activity beyond the deep cortical layers, in the underlying white matter $(n=3)$. Figure 4 shows such data, with activities recorded by a ring placed on the cortical surface and by means of a microelectrode, penetrating through the ring, at different depths until the white matter. Clear-cut fast waves at various depths in the cortical mantle hecame barely visible in the white matter, the reduction in the amplitude of fast waves exceeding $70 \%$ from a depth of $0.9 \mathrm{~mm}$ to the white matter $(2.5 \mathrm{~mm})$.

Moreover, action potentials crowned the negative components of local fast waves in tracks exploring various cortical depths from $0.15-0.2 \mathrm{~mm}$ to $1.5 \mathrm{~mm}(n=6)$. Figure 5 depicts two locations of the microelectrode, the first at $\sim 0.15 \mathrm{~mm}$ where the slow oscillation was not yet reversed $(A)$ and the second at a depth of $0.7 \mathrm{~mm}$ where the long-lasting surface-negative wave was reversed into a depth positivity $(B)$. At both levels, neuronal discharges were rhythmically suppressed during the prolonged surface negativity of the slow oscillation (see expanded insets). Fast activities ( $\sim 40$ $\mathrm{Hz}$ ) were typically reduced, up to their disappearance, during the surface-negative (depth-positive) waves (see filtered traces, 15-80 $\mathrm{IIz}$, in Fig. $5 B$ ). Unit discharges werc consistently associated with focal negative waves at depths of $0.15 \mathrm{~mm}$ and $0.7 \mathrm{~mm}$, and spike-triggered averages revealed in-phase fast activity at the surface and at 0.15 or $0.7 \mathrm{~mm}$, with surface waves lagging behind 

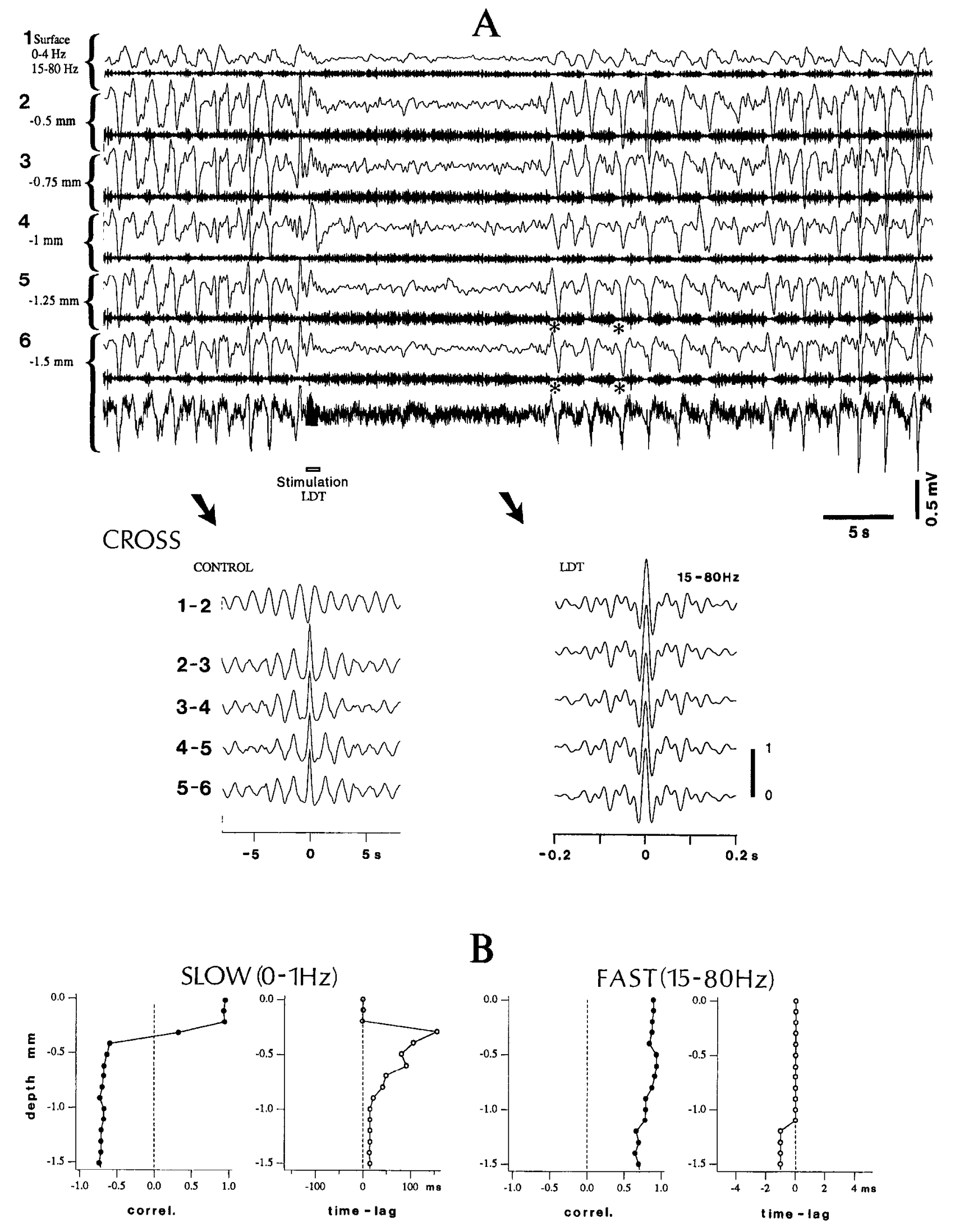

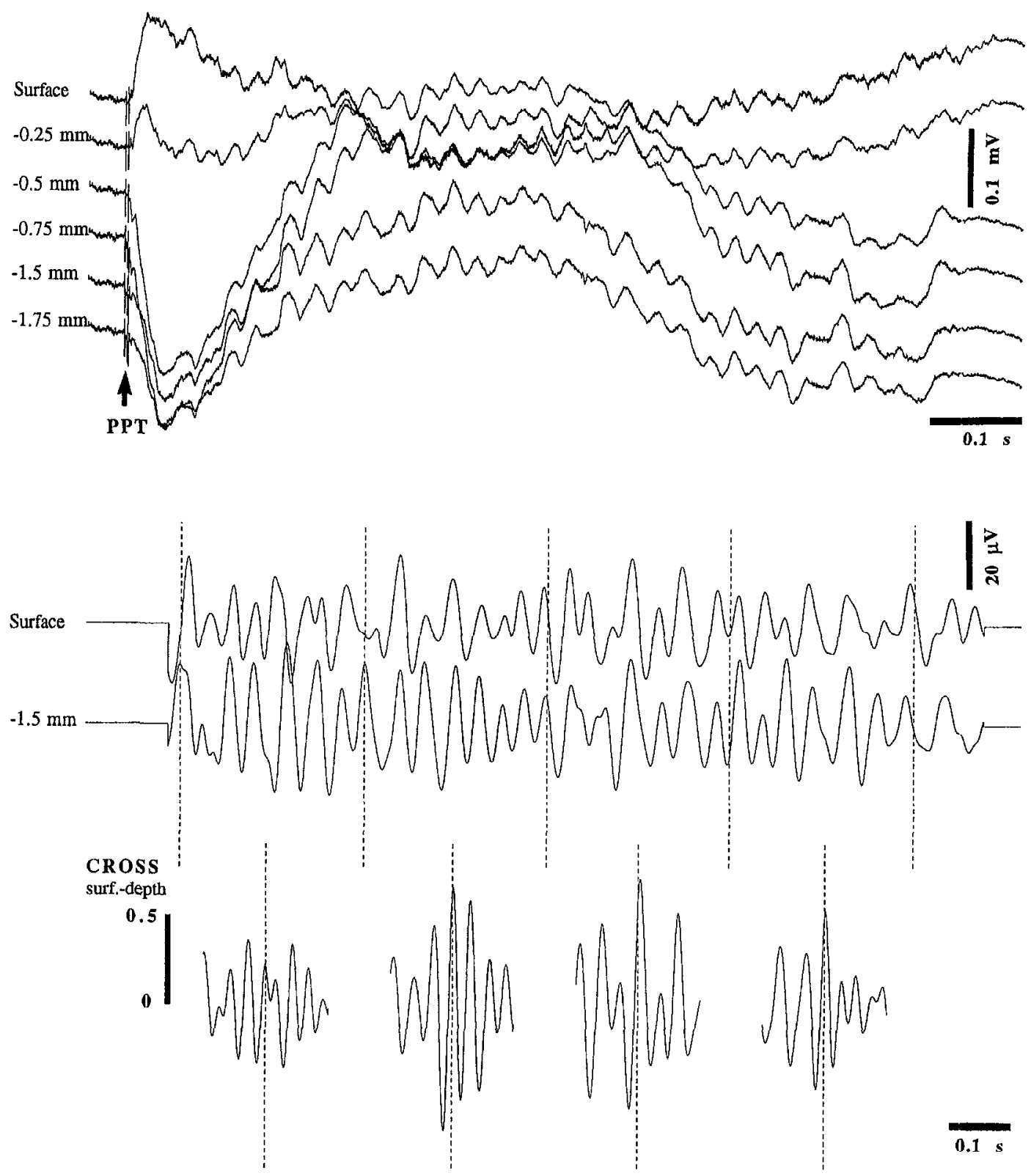

Figure 3. PPT-induced coherent fast oscillations $(35-40 \mathrm{~Hz})$ at the surface and at various cortical depths of area 5. Ketamine and xylazine anesthesia. Top, Averaged responses $(n=15)$ to two short delayed $(3 \mathrm{msec})$ shocks applied to the PPT nucleus. The response started with a surface-positive (depth-negative) wave lasting for $\sim 60 \mathrm{msec}$, followed by fast waves that were in-phase at the surface and depth leads. Bottom, The surface- and depth $(1.5 \mathrm{~mm})$-evoked potentials were filtered $(15-80 \mathrm{~Hz})$ and cross-correlated (CKOSS) within four windows (1-4) of $300 \mathrm{msec}$. Note in-phase relations in windows $2-4$.

Figure 2. Contrast between the depth-reversal of slow oscillation $(0.7 \mathrm{~Hz})$ and in-phase correlation of fast activity $(35-40 \mathrm{~Hz})$. Ketamine and xylazine anesthesia. $A$, Simultaneous recording at the surface and at five different depths through a six-array electrode inserted in precruciate area 6 . The top panel shows the slow oscillation and its suppression by a pulse-train to the LDT $(300 \mathrm{~Hz}, 1 \mathrm{sec})$ simultaneously with the enhanced amplitudes of fast waves after LDT stimulation. T'he slow oscillation resumed $17 \mathrm{sec}$ after LDT stimulation. Traces were digitally filtered once between 0 and $4 \mathrm{~Hz}$ (upper trace in each channel) and another time between 15 and $80 \mathrm{~Hz}$ (lower trace). The original data from the bottom channel are added below. Two periods before and after LDT stimulation, indicated by arrows, are expanded in the bottom panels. Cross-correlations (CROSS) were computed from two 12 sec periods, before and after LDT stimulation. Cross-correlations between nonfiltered successive channels during the prestimulation period (at left, CONTROL) show highly coherent slow oscillation, with reversal between leads 1 (surface) and $2(-0.5 \mathrm{~mm}$; the upper CROSS has the central peak downward; the time lag between $1-2$ is $0.1 \mathrm{sec}$ ). The cross-correlations between the same channels filtered at $15-80 \mathrm{~Hz}$. (at right, LDT) demonstrate in-phase fast activities at all depths. The central peaks resulting from cross-correlations between nonfiltered activity (compare with prestimulation period) are attrilsutable to incoherent slow oscillations that survive during this activated epoch (see top panel). $B$, Depth profile of slow $(0-1 \mathrm{~Hz})$ and fast oscillations $(15-80 \mathrm{~Hz})$ by means of a tungsten microelectrode penetrating the cortical area 5 through a ring placed at the surface in successive steps of $0.1 \mathrm{~mm}$. Note reversal of slow oscillation between 0.2 and $0.3 \mathrm{~mm}$, whereas the fast activity did not reverse down to the deepest cortical layer. 

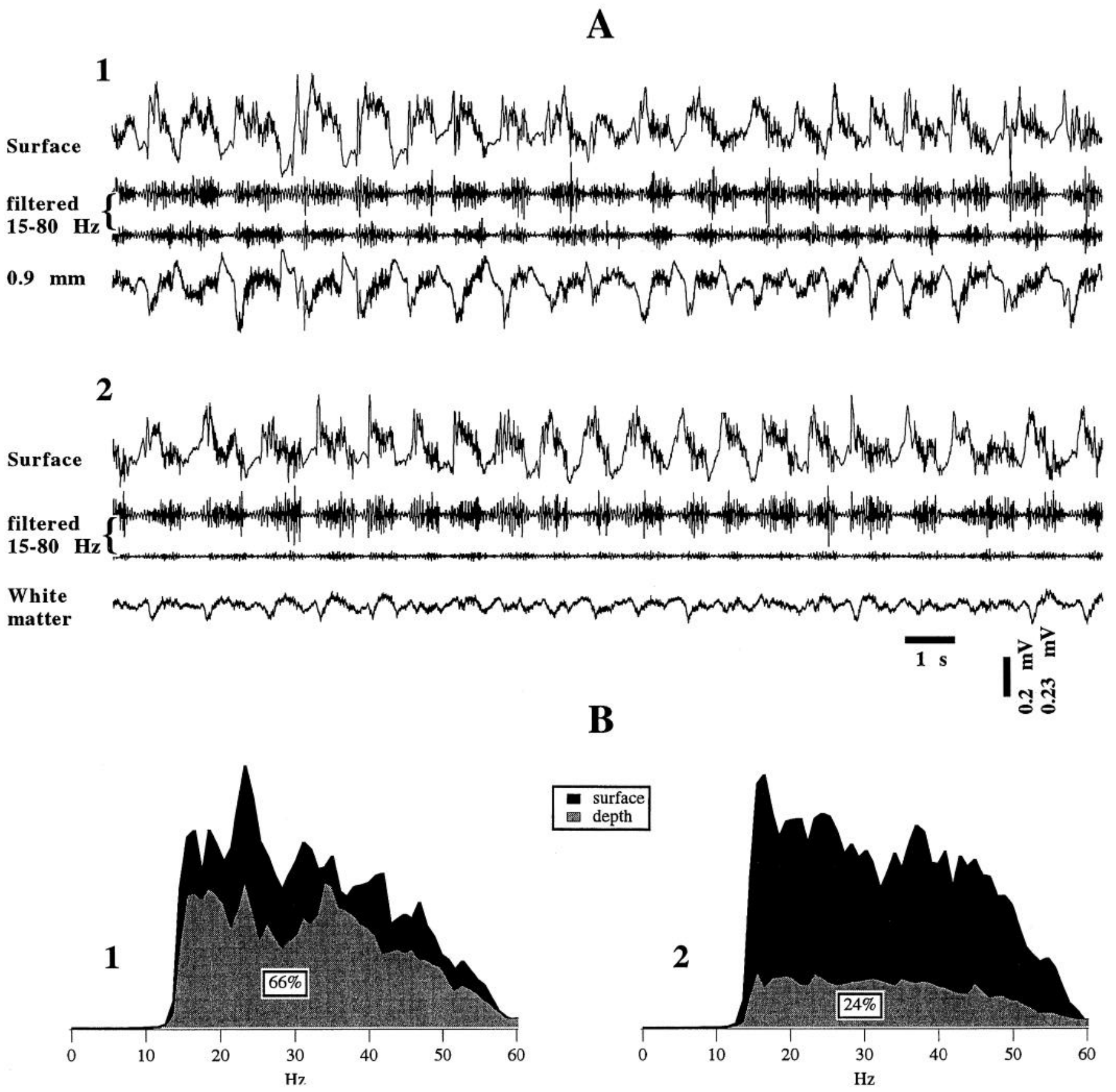

Figure 4. The amplitude of fast cortical oscillation is strikingly reduced in the white matter. Ketamine and xylazine anesthesia. $A$, Simultaneous recordings by a ring placed on the surface of area 5 and a tungsten microelectrode penetrating through the ring, first at a depth of $0.9 \mathrm{~mm}(l)$ and thereafter at a depth of $2.5 \mathrm{~mm}$ in the white matter (2). Between the original activities from the surface and cortical depth or white matter, filtered fast activities $(15-80 \mathrm{~Hz}$ ) from both channels. B. Power spectrum of fast activities showing waves at the surface (black) and in the depth or white matter (gray), corresponding to recordings in $A 1$ and $A 2$. The area of power spectrum from depth-recorded $(0.9 \mathrm{~mm})$ waves was $66 \%$ of the area from surface-recorded activity, whereas in the white matter the area represented only $24 \%$ from surface-recorded activities. Filtered fast activities are enhanced (see different calibrations). In this and in other figures with power spectra (Figs. 7, 12), same ordinate scaling in different panels comparing different epochs.

the depth waves by $\sim 2-3$ msec. The superficial location (above 0.2 $\mathrm{mm}$ ) of the microelectrode in Figure $5 \mathrm{~A}$ is also indicated by the lack of reversal of slow oscillation, which occurs below $0.25 \mathrm{~mm}$ (see Figs. 1, 2). This result indicates that the focal negative field potentials of the fast oscillation are associated with action potentials throughout the cortical depth and that they occur in-phase with the fast waves recorded from the cortical surface (see also Fig. 8, with intracellular recordings).
Of 76 tracks in various areas, in-phase fast activities, from the cortical surface to the deepest layers, were found in 69 descents $(90 \%)$. In the remaining seven tracks, we found a circumscribed zone in the cortical depth $(0.8-1 \mathrm{~mm})$ where the fast waves were reversed with respect to those recorded from the surface. Five of those seven tracks were in the lateral part of the precruciate area 4 (see Discussion). The intracellular recording of the area 4 neuron in Figure $6 A$ shows the suppression of slow oscillation ( 0.6 
$\mathrm{Hz}$ ) and the activation patterns elicited by a pulse train to the mesopontine PPT nucleus. The expanded EEG and intracellular traces during the period immediately after the cessation of PPT stimulation (arrow) demonstrate that the discharges of the cells were generally related to depth-negative (surface-positive) field potentials. Moreover, spike-triggered averages (action potentials at time 0 ) and wave-triggered averages (peak negativity of depth field potentials at time 0 ) demonstrate the phase-opposition of fast oscillatory waves recorded from the cortical surface and depth (Fig. 6B).

\section{Fast oscillations depend on the depolarization of cortical neurons}

Data presented above showed the selective disruption of fast rhythms during the prolonged depth-positive cortical EEG waves (Figs, $1,2 A, 5 B$ ) that are known to be associated with prolonged hyperpolarizations in cortical and thalamic cells (Steriade et al., 1994; Contreras and Steriade, 1995).

Intracellular recordings were performed to determine the dependence of fast EEG rhythms on the depolarization of cortical neurons, either elicited by stimulation of mesopontine cholinergic nuclei or occurring spontaneously for brief epochs. Multisite recordings of surface and depth EEG waves, extracellular unit discharges and field potentials recorded through the same microelectrode, and intracellular activities from an adjacent cortical area $(n=28)$ invariably showed that during a brief period of activation when the slow oscillation was reduced or abolished, the neurons displayed a sustained depolarization in conjunction with an enhancement of fast EEG waves at 30-40 Hz. The rhythmic depolarizing envelopes of the neuron depicted in Figure $7 \mathrm{~A}$ had durations of $0.6-0.7 \mathrm{sec}$, and the subsequent hyperpolarizations lasted for $0.5-0.6 \mathrm{sec}$ during the slow oscillation at $\sim 0.8 \mathrm{~Hz}$, whereas the $V_{\mathrm{m}}$ was no longer interrupted by rhythmic hyperpolarizations during the whole $6 \mathrm{sec}$ period of spontaneous activation. During the activated epoch, the power spectra of EEG waves in the $15-50 \mathrm{~Hz}$ frequency band increased by $>90 \%$ compared with those of control periods (Fig. $7 B$ ). This difference resulted from the fact that fast EEG waves were continuous during the activated epoch (simultaneously with the sustained depolarization of the neuron), whereas fast activities were periodically suppressed during the slow oscillation. Similar aspects were observed with intracellularly recorded neurons from other areas.

The fast oscillations in intracellularly recorded neurons, their occurrence during the depolarizing phases of the slow oscillation, and their relations to surface- and depth-FFG waves were analyzed by using auto- and cross-correlation analyses as well as spike- or wave-triggered averages $(n=17)$. Similar results were obtained in association areas 5 and 7, primary somatosensory areas, and motor area 4 . These data are exemplified by Figure 8 , with recordings from SI cortex. Typically the prolonged cellular hyperpolarizations were accompanied by the long-lasting depthpositive EEG waves, whereas the depolarizations started in close time relation with a sharp depth-negative EEG potential, ultimately leading to fast activity. The filtered EEG and cellular traces in Figure $8 A$ (see especially the second of three slow oscillatory cycles) show that the depth-negative EEG activity and neuronal depolarization initiated a few waves at the spindle frequency $(10 \mathrm{~Hz})$ and reached higher frequencies $(40 \mathrm{~Hz})$ toward the middle part of the depolarizing phase. Figure $8 B$ (from the third slow oscillatory cycle) is expanded below and shows in-phase fast EEG waves at the surface and at depth (first asterisk) on the one hand and phase-opposition between intracellular depolariz- ing wavelets and depth-negative field potentials (second asterisk) on the other. These relations were preserved with wave-triggered averages (Fig. $8 C$; time 0 is the peak negativity of depth EEG) showing a rhythm with a frequency of $40 \mathrm{~Hz}$ in the surface- and depth-EEG, an in-phase fast oscillation between the surface- and depth-EEG, and cell depolarization, lagging the peak negativity of depth field potential by $5-8 \mathrm{msec}$.

The fast activity extended throughout the depolarizing phase of the slow oscillation and was particularly well expressed in those few instances in which presumably dendritic recordings were performed $(n=3)$. Such recordings were hypothesized as dendritic because of the incongruity between the resting $V_{\mathrm{m}}$ (more negative than $-70 \mathrm{mV})$ and the height of action potentials $(<40$ $\mathrm{mV}$ ); marked differences between the spike trigger level and $V_{\mathrm{m}}$ values; spikes with varying amplitudes suggesting that they arose from several dendritic hot spots; doublets or triplets with very short interspike intervals (1-3 msec); and long-term recordings (>80 min in Fig. 9), thus precluding signs of injury. These neurons exhibited ample oscillations of the membrane throughout the depolarizing envelope of the slow oscillation, reaching frequencies of $35-40 \mathrm{~Hz}$ and giving rise to single spikes or spike doublets (Fig. 9).

Fast oscillations could also be elicited by short or long depolarizing current pulses $(n=18$; i.e., $20 \%$ of tested neurons in motor area 4 , somatosensory area $3 b$, and association area 5). The basic features exhibited by responses to depolarizing current pulses are as follows. (1) In some of these neurons $(n=9)$, spike-bursts (with an intraburst frequency of 200-400 $\mathrm{Hz}$ ) recurred at a frequency of $30-40 \mathrm{~Hz}$. when the pulse adequately depolarized the membrane, whereas at more negative levels of $V_{\mathrm{m}}$, only a passive response was seen. In the neuron depicted in Figure $10 A$, the activity triggered by depolarizing pulses was initiated by spike doublets or triplets, but afterward the rhythmic spike doublets at $30 \mathrm{~Hz}$ were highly stereotyped (see traces $a-d$ ). (2) The remaining neurons responded similarly, with spike doublets or triplets at $40 \mathrm{~Hz}$ or even higher frequencies (up to $80 \mathrm{~Hz}$ ); however, by diminishing the intensity of the depolarizing pulse, rhythmic bursting was replaced by enhanced amplitudes of background PSPs (Fig. $10 B, C$ ), instead of by a purely passive response. This suggested that the propensity of some cortical neurons to display tast oscillations depends on combined factors, i.e., their intrinsic properties boosted by network synaptic activity.

To test this possibility, we applied depolarizing current pulses with the same parameters during epochs with poor or null synaptic activity, compared with epochs in which the network activity is high. It is known that during the long-lasting hyperpolarizations of the slow oscillation (related to prolonged depth-positive EEG waves), PSPs are strikingly diminished, whereas the subsequent depolarizing phases are accompanied by increased synaptic activities (Fig. 11 $A$ ) (Steriade et al., 1993d; Contreras and Steriade, 1995). During the hyperpolarizing phases of the slow oscillation, long depolarizing pulses ( $400-500 \mathrm{msec}$ ) elicited a slowly adapting response. The action potentials were followed by a fast afterhyperpolarization and a medium after-hyperpolarization, and the adaptation occurred in parallel with an increase in the amplitude and duration of both after hyperpolarizations (Fig. 11BI). This aspect, betraying the intrinsic properties of a subclass of regularspiking cortical pyramidal neurons, was described previously in vitro (Stafstrom et al., 1984; Schwindt et al., 1988) and in vivo (Nuñez et al., 1993). At variance, the action potentials elicited by current pulses during the depolarizing phases of the slow oscilla- 


\section{A}

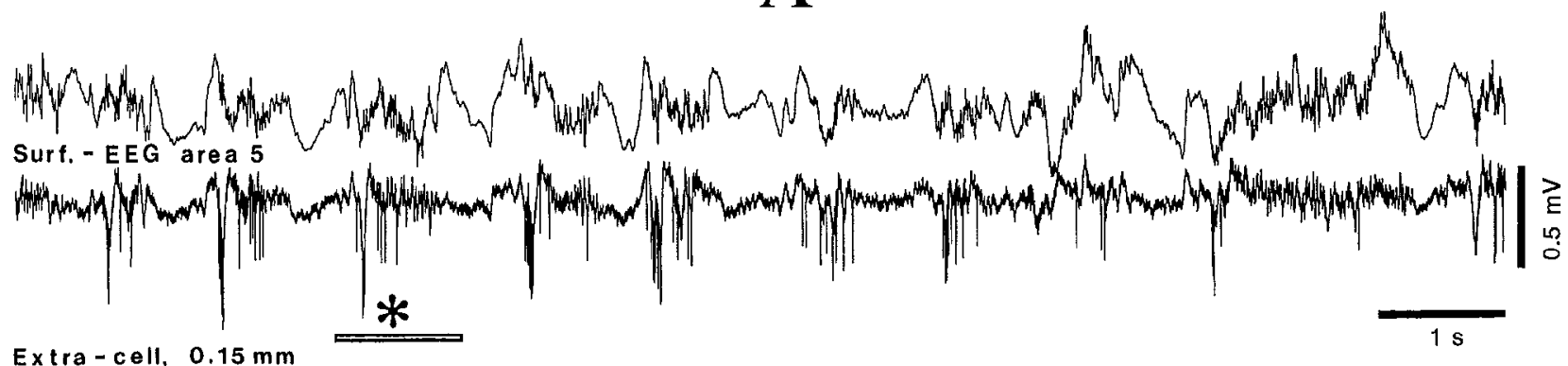

Extra-cell, $0.15 \mathrm{~mm}$

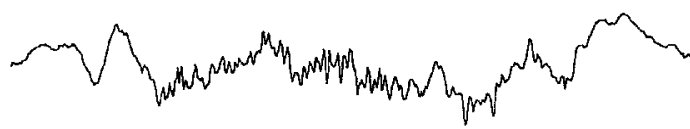

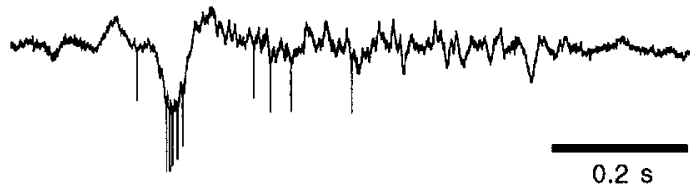

STA

B

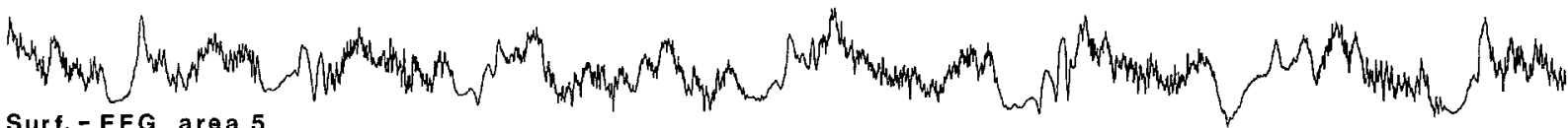
Surf. - EEG area 5
Extra-cell, $0.7 \mathrm{~mm}$

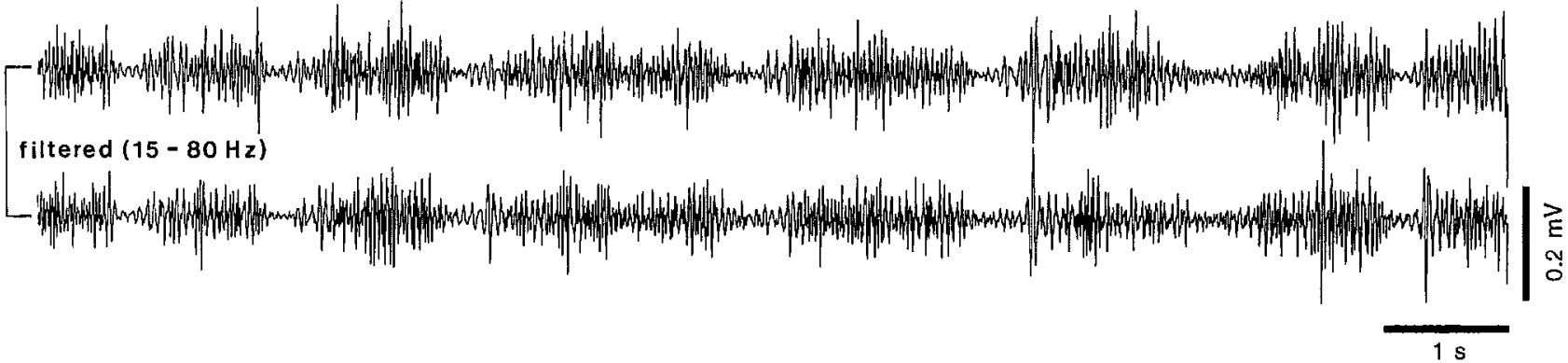

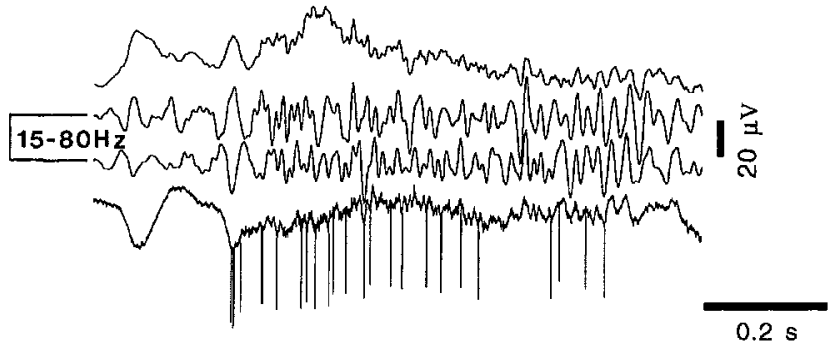

STA

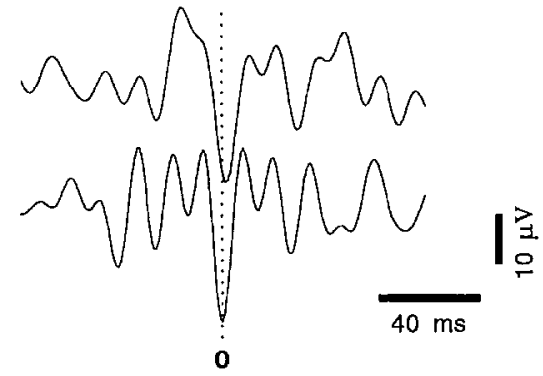




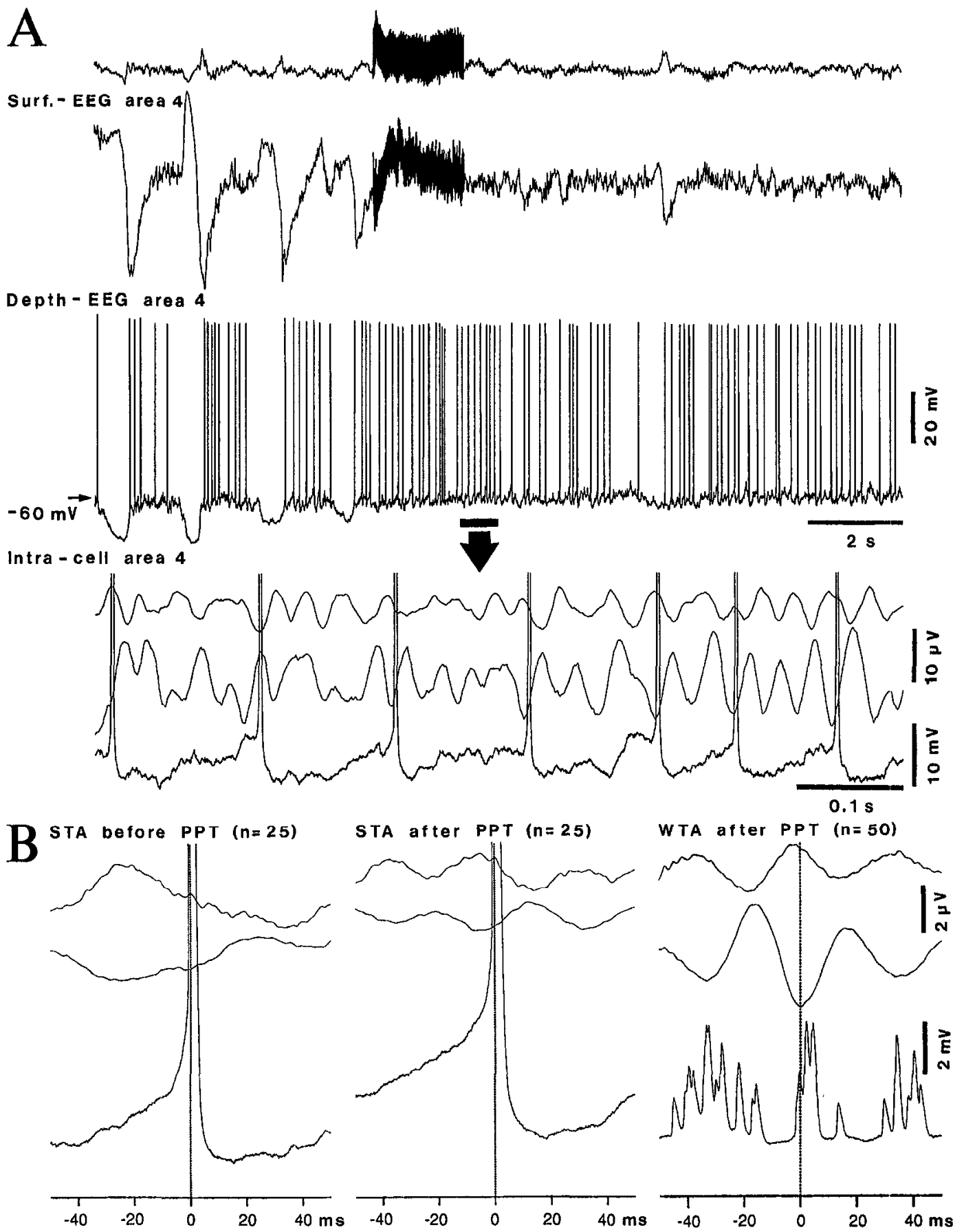

Figure 6. Phase-opposition between fast $(\sim 30 \mathrm{~Hz})$ surface- and depth-EEG wave. Ketamine and xylazine anesthesia. Intracellular recording of regular-spiking cell at a depth of $1.2 \mathrm{~mm}$ in area 4 , simultaneously with recording of surface- and depth-EEG waves through coaxial electrode in area $4,1 \mathrm{~mm}$ apart from the micropipette. A, Slow oscillation $(0.6 \mathrm{~Hz})$ and activation patterns elicited by $2 \mathrm{sec}$ pulse train $(300 \mathrm{~Hz})$ to the brainstem PPT nucleus. Part marked by horizontal bar on the intracellular trace (arrow) is expanded and amplified below. Note that most of the action potentials are related to the depth-negative field potentials, which are in phase opposition to surface-EEG waves (see the last five of seven spikes), but variant temporal relations are seen for the first two spikes. $B$, Spike-triggered averages (STA) during the periods before and after PPT stimulation (action potentials of intracellularly recorded ncuron werc used to trigger surface- and depth-EEG waves) and wave-triggered averages (WTA) after PPT stimulation (the peaks of depth-negative field potentials were used to trigger the surface-EEG waves and intracellular activities). Note phase-opposition (surface-depth) between fast oscillations after PPT stimulation.

Figure 5. Focal negative fast waves $(\sim 40 \mathrm{~Hz})$ are crowned by action potentials at hoth superficial and deep cortical layers. Ketamine and xylazine anesthesia. $A$, Simultaneous recording through ring placed on the surface and through microelectrode picking up local field potential and unit discharges at $0.15 \mathrm{~mm}$ from surface. Period marked by horizontal bar (asterisk) is expanded below. At right, spike-triggered average (STA); 50 sweeps with waves at the surface and $0.15 \mathrm{~mm}, 80 \mathrm{msec}$ before and after the action potentials. $B$, Same track with microelectrode at a depth of $0.7 \mathrm{~mm}$. Below the two original traces, activity was filtered between 15 and $80 \mathrm{~Hz}$ to show reduction or disappearance of fast waves during the depth-positive component of the slow oscillation. Part marked by horizontal bar (asterisk) is expanded below (left). The top and bottom traces represent the nonfiltered traces from the surface and depth, and the middle traces represent the same waves, but after filtering $(15-80 \mathrm{~Hz})$ and increasing their amplitudes to show $40 \mathrm{~Hz}$ activity. At right, STA (50 sweeps, as in $A$ ), showing in-phase relation, with the surface lagging the depth by 2-3 msec, same as in $A$. 


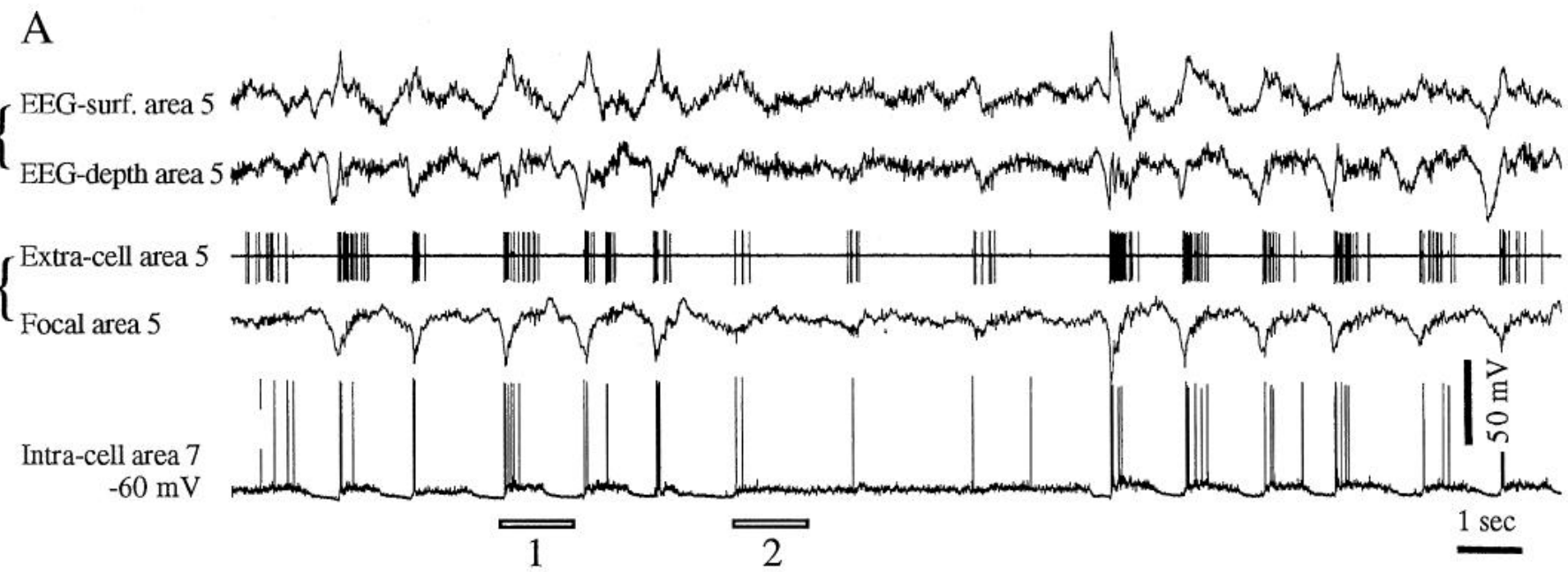

EEG-surf. W filtr. $15-50 \mathrm{~Hz}$

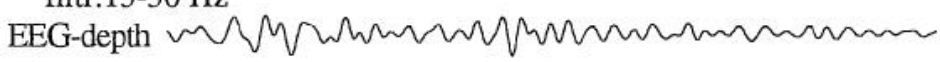

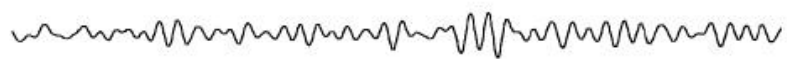

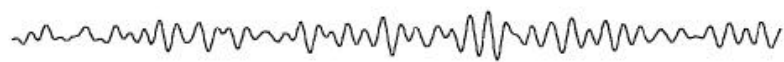
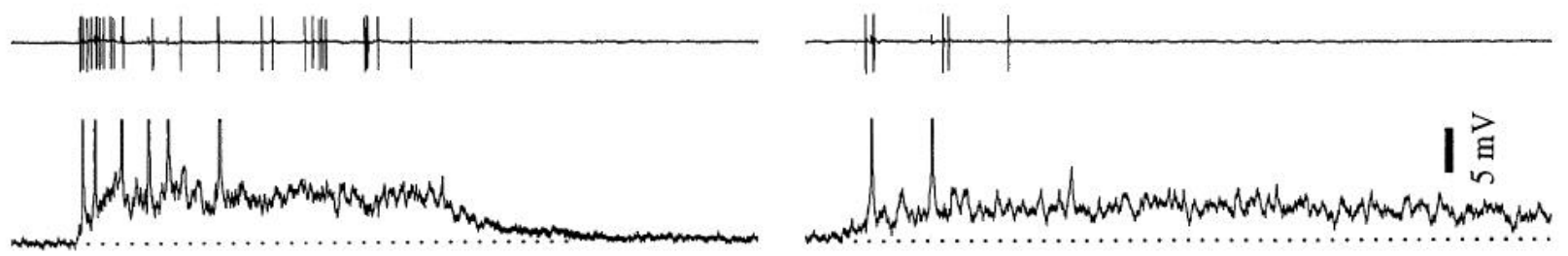

$0.2 \mathrm{sec}$

B

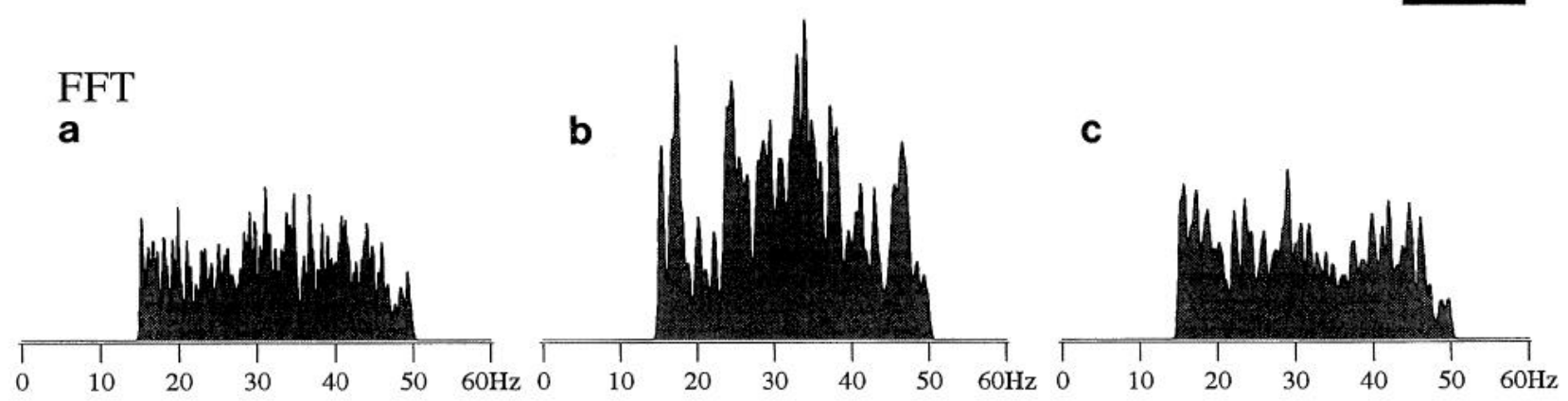

Figure 7. Increased power of fast waves $(15-50 \mathrm{~Hz}$, peak around $35 \mathrm{~Hz})$ during spontaneous epoch of EEG activation associated with sustained depolarization of cortical cell. Ketamine and xylazine anesthesia. A, Multisite recording of surface- and depth-EEG from area 5, extracellular unit activity and local field potentials (through the same microelectrode) from area 5, and intracellular activity of area 7 neuron. Parts 1 (during slow oscillation) and 2 (onset of shortly activated epoch) are expanded below, depicting EEG waves filtered between $15 \mathrm{and} 50 \mathrm{~Hz}$, extracellular discharges of area 5 cell, and intracellular activity of area 7 neuron. In 2, note sustained depolarization of area 7 neuron (dotted line tentatively indicates the baseline) and in-phase fast EEG waves recorded from the surface and depth of area 5. B. Surfaces of FFTs from area 5 EEG waves (filtered between 15 and $50 \mathrm{~Hz}$ ) in a control period before activation $(a)$, during activation $(b)$, and during a postactivation period $(c)$. The power spectrum in the $15-50 \mathrm{~Hz}$ band $($ peak around 35 $\mathrm{Hz}$ ) was enhanced by $93 \%$, compared with the preactivation period, because of the absence of prolonged inhibitory phases of the slow oscillation.

tion were continuously boosted by the background synaptic activity and were grouped within spike sequences with a frequency of $30-40 \mathrm{~Hz}$ (Fig. 11B2). The hyperpolarizing pulses similarly reflected the contrasting background activities of these two periods (Fig. 11B).

\section{Short-range intracortical coherence of fast oscillations}

The synchronization of fast rhythms within the same cortical area is demonstrated by cross-correlations between surface and depth activities in sensory, motor, and association areas (Figs. 1-3, 8).
We asked whether the coherence of fast oscillation also appears among adjacent and more distantly located cortical areas. The results of these experiments indicate that distinctly from the long-range synchronization of the sleep slow $(<1 \mathrm{~Hz})$ oscillation that appears among close as well as distant cortical areas (Amzica and Steriade, 1995a,b), the coherence of fast rhythms is spatially limited.

As shown above (Figs. 1,2), stimulation of mesopontine cholinergic nuclei blocked the slow oscillation and elicited fast rhythms. Cross-correlation and FFT analyses of field potentials 


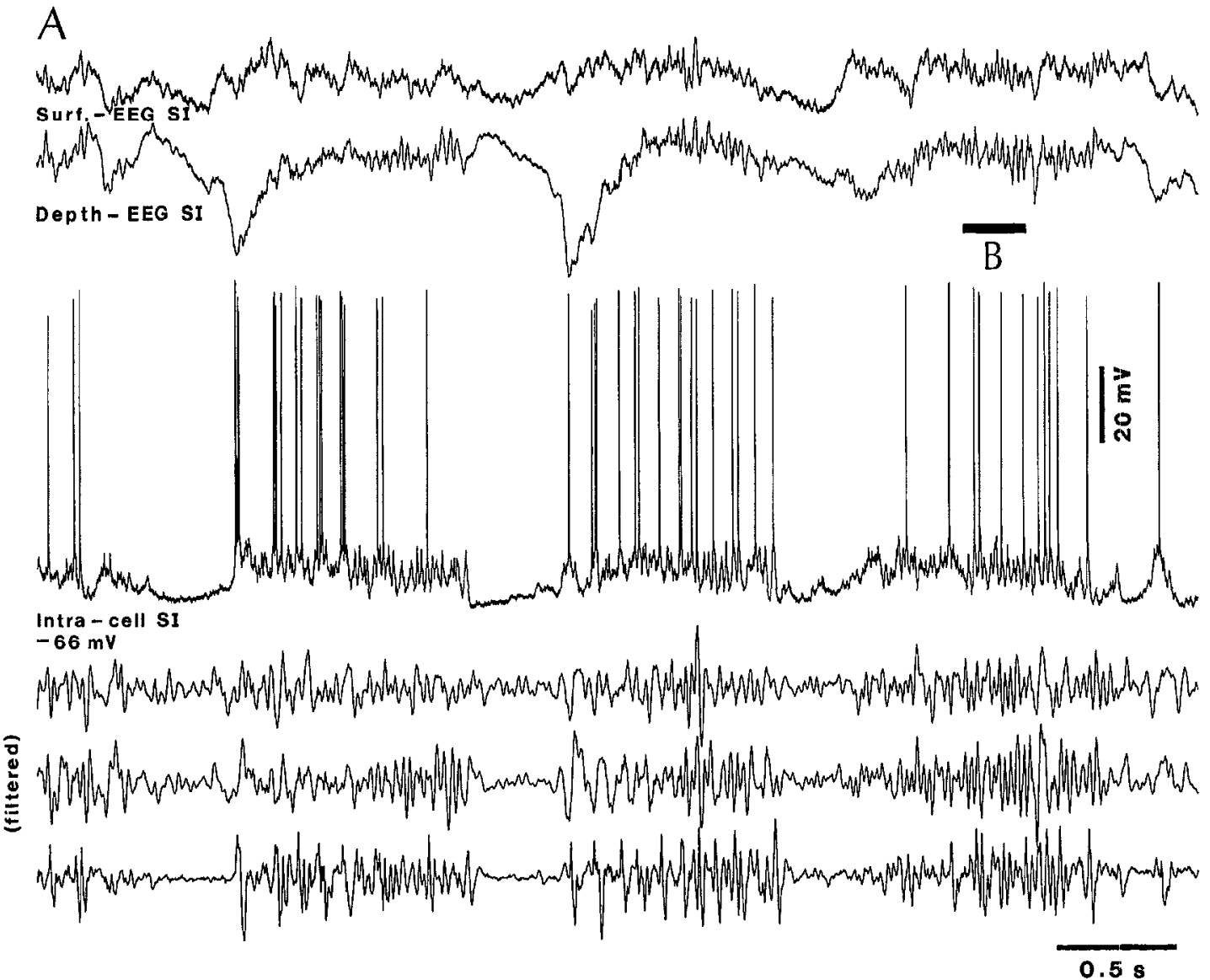

B

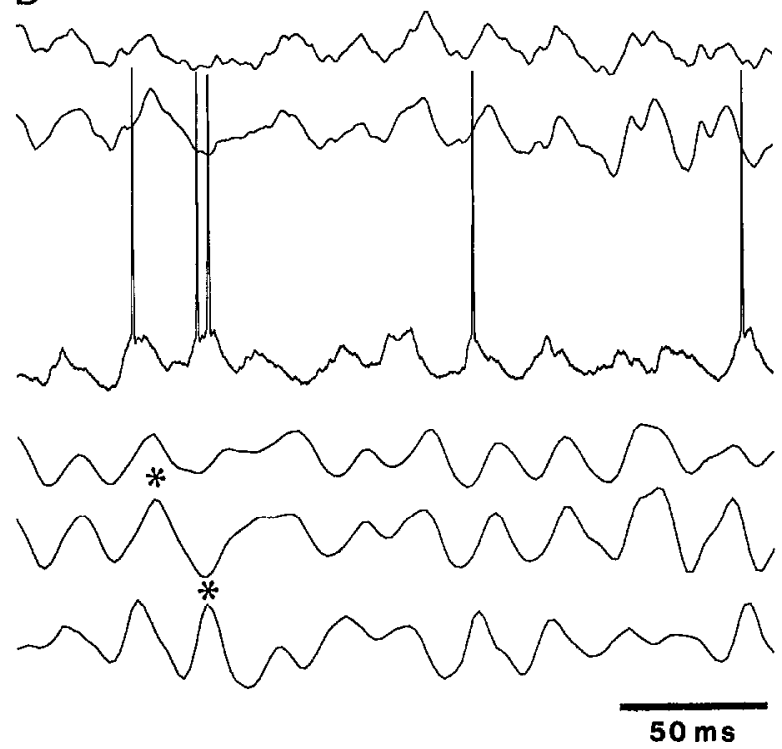

C

Wave-triggered-average

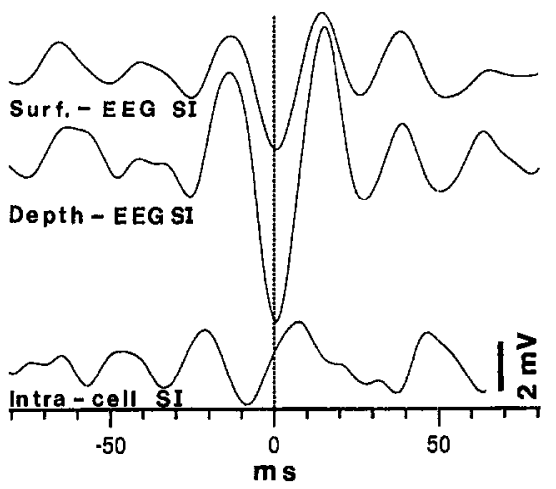

Figure 8. Fast activity $(40 \mathrm{~Hz})$ in cortical neurons occurs during the depolarizing phases and is suppressed during the prolonged hyperpolarizations of slow oscillation $(0.6-0.7 \mathrm{~Hz})$. Ketamine and xylazine anesthesia. Cell at a depth of $0.3 \mathrm{~mm}$ in SI cortex, with fast spike $(0.8 \mathrm{msec})$ and after-depolarizing potential occasionally giving rise to doublets $(3 \mathrm{msec}$ delayed spikes). $A$, Slow EEG oscillation recorded at the surface and depth of SI cortex, simultaneously with intracellular recording from SI neuron. Below, three traces depict the same activities (in the same order), but filtered between 10 and $100 \mathrm{~Hz}$. The second cycle of the slow oscillation shows that the activities following the sharp depth-negative deflection of the slow oscillation are initiated by EEG waves and intracellular potentials within the frequency range of spindles $(10 \mathrm{~Hz}$ ), thereafter followed by fast activity ( $40 \mathrm{~Hz}$ ). The part marked by $B$ is extended below, in $B$, to demonstrate in-phase, surface- and depth-EEG fast activity during the depolarizing phase of the slow oscillation (left asterisk) and phase-opposition between depth-EEG and intracellular activity (right asterisk). $C$, Wave-triggered averages (time 0 is the peak negativity of depth-negative field potential). Note fast activity $(40 \mathrm{~Hz})$ and depolarization of cell, lagging by $5-8 \mathrm{msec}$ the peak of depth-negative field potential. 

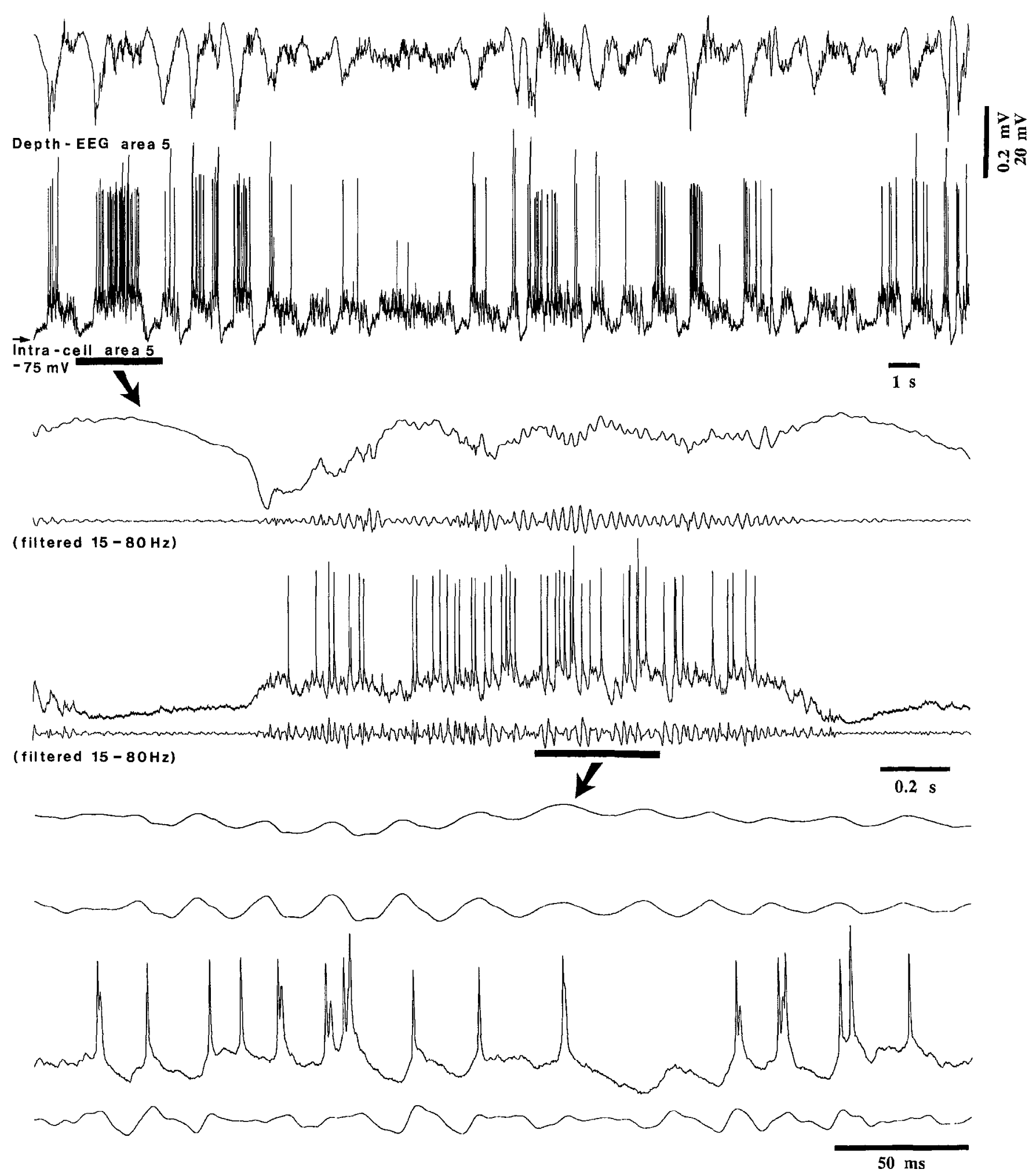

Figure 9. Fast activity $(35-40 \mathrm{~Hz})$ during the depolarizing phase of slow oscillation in a presumed dendritic recording (see text). Ketamine and xylazine anesthesia. Intracellular recording of neuron at a depth of $0.5 \mathrm{~mm}$ in area 5. Part marked by horizontal bar in the top panel is expanded below (arrow), where below depth-EEG and intracellular traces there is also the filtered activity ( 15 to $80 \mathrm{~Hz}$ ). Part marked by horizontal bar in the middle panel is further expanded below (arrow).

simultaneously recorded from scvcral cortical foci during the sleep-like patterns preceding the brainstem PPT stimulation and during the activated patterns outlasting the PPT pulse-train (Fig. 12) showed the following: (1) low-frequency (mainly $<5 \mathrm{~Hz}$ ) rhythms during the control period and synchronization of the slow cortical oscillation $(0.4 \mathrm{~Hz})$ before PPT stimulation; (2) strong reduction in the low-frequency rhythms, appearance of fast waves (mainly between 30 and $40 \mathrm{~Hz}$ ), and intracortical coherence of 

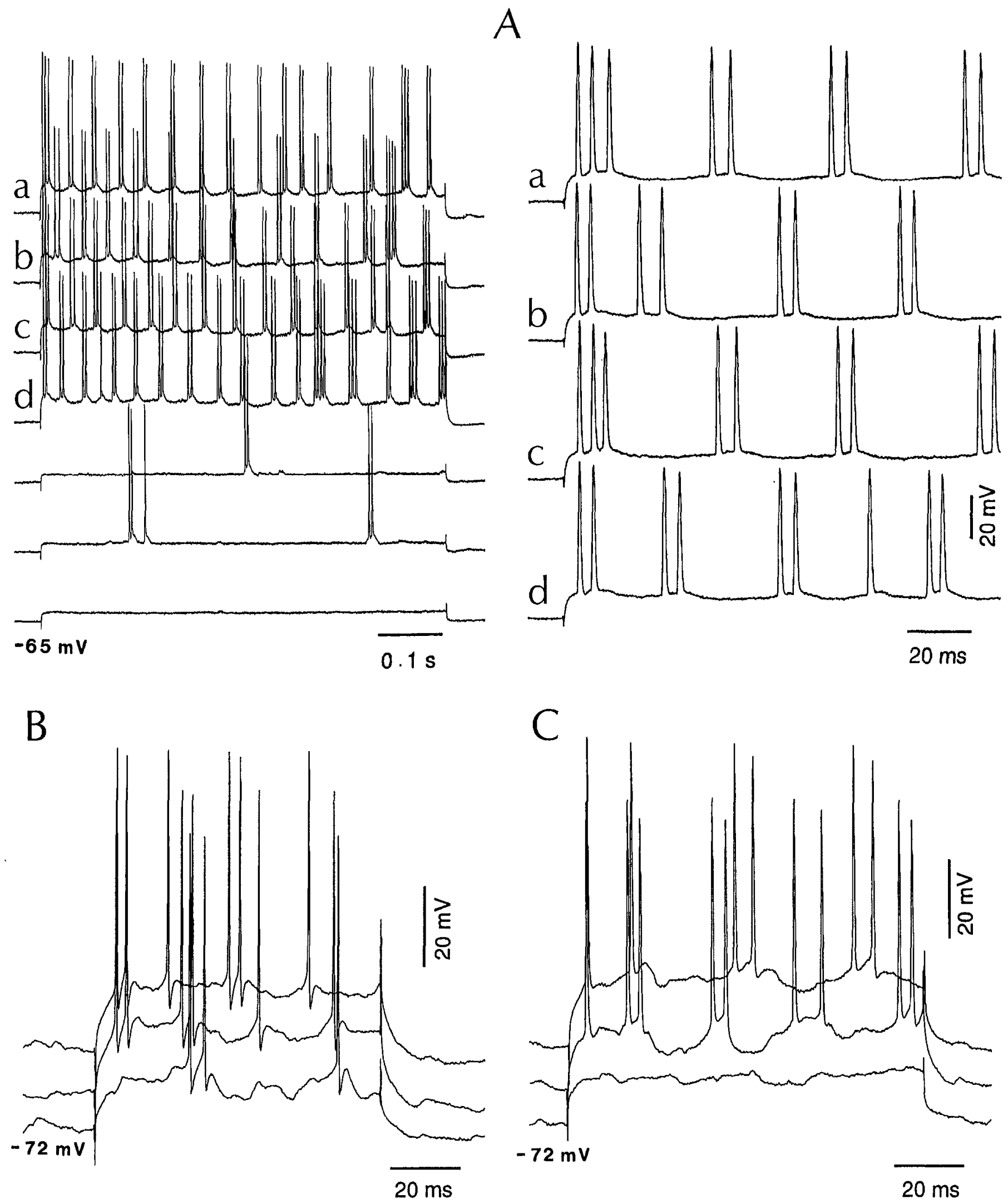

Figure 10. Fast rhythmic spike bursts elicited in cortical neurons by depolarizing current pulses. Intracellular recordings of three different neurons under ketamine and xylazine anesthesia. $A$, Neuron at a depth of $0.4 \mathrm{~mm}$ in anterior suprasylvian area 5 . Depolarizing currents of $600 \mathrm{msec}, 0.5 \mathrm{nA}$ in $a-d$, and $0.2 \mathrm{nA}$ in the botlom three traces. At right, expanded traces of $a-d$. Note, in $a-d$, spike doublets and triplets (interspike intervals: 4 msec) recurring at $\sim 30$ Hz. $B$, Neuron at a depth of $0.8 \mathrm{~mm}$ in SI. Depolarizing current pulses (duration $80 \mathrm{msec}$ ) with increasing amplitudes $(0.7,0.8$, and $1 \mathrm{nA}$ ) applied at the same $V_{\mathrm{m}}$. Pulses of 0.8 and $1 \mathrm{nA}$ elicited spike doublets at $50-80 \mathrm{~Hz}$. $C$, Neuron at a depth of $0.5 \mathrm{~mm}$ in SI. Depolarizing current pulses (duration 100 $\mathrm{msec}$ ) with increasing amplitudes $(0.6,0.7$, and $1.1 \mathrm{nA})$ at the same $V_{\mathrm{m}}$. Pulses of 0.7 and $1.1 \mathrm{nA}$ elicited 3-4 spike doublets at $\sim 40 \mathrm{~Hz}$. 

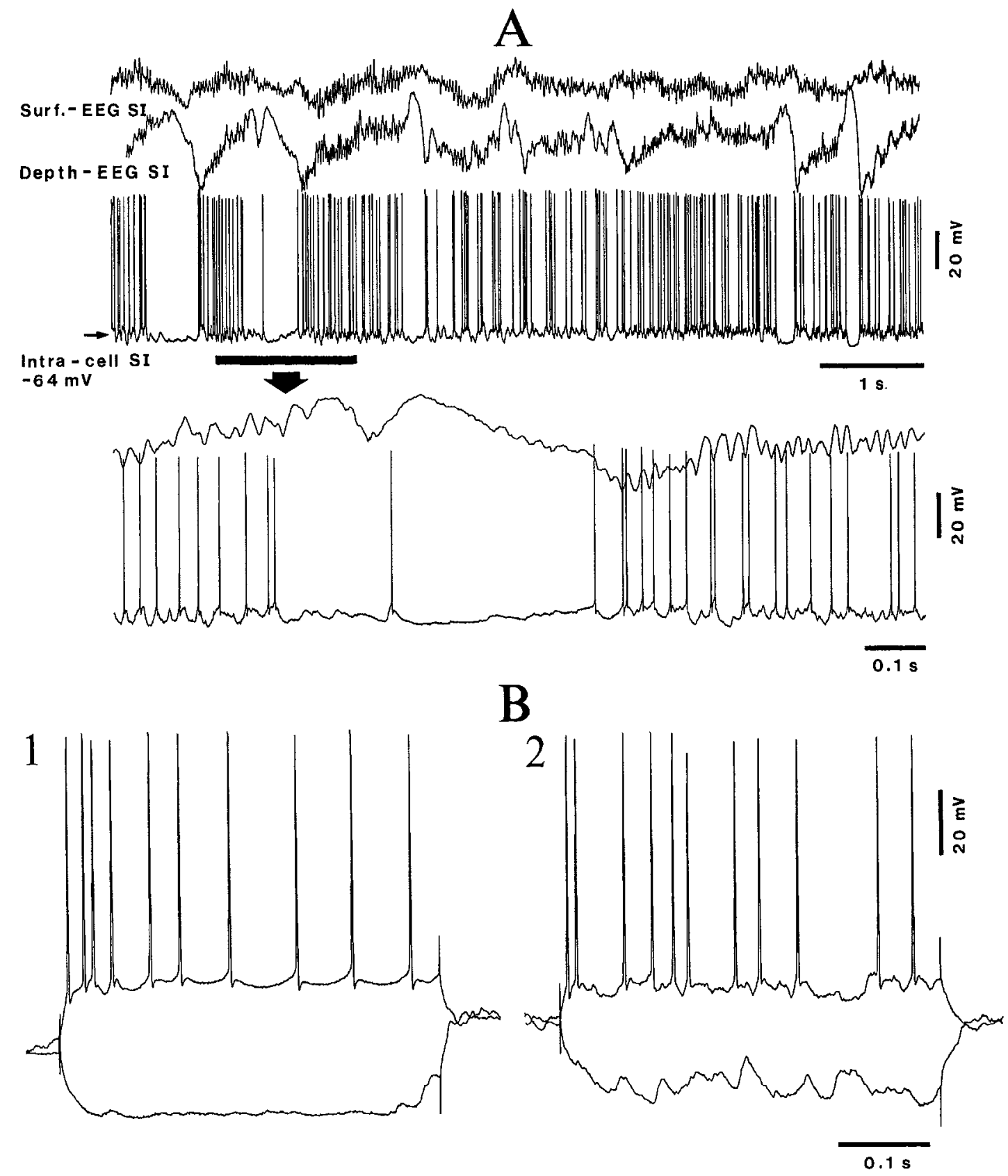

Figure 11. Effects of depolarizing current pulses during epochs of reduced or null synaptic activity, compared with epochs with intense synaptic activity. Intracellular recording of regular-spiking neuron in SI (depth $0.8 \mathrm{~mm}$ ) under ketamine and xylazine anesthesia. $A$, Strikingly reduced synaptic activity during the prolonged hyperpolarizing phases of the slow oscillation associated with depth-positive EEG waves and increased synaptic activity during the fast activity $(40-50 \mathrm{~Hz})$ that follows the sharp depth-negative EEG deflection (part marked by horizontal bar is expanded below; arrow). $B$, Differential responses to depolarizing and hyperpolarizing current pulses $(1 \mathrm{nA})$ applied during the prolonged hyperpolarizations of the spontaneous slow oscillation associated with reduced synaptic activities $(l)$ and during periods with increased synaptic activities (2).

Figure 12. Intracortical coherence of slow $(0.4 \mathrm{~Hz})$ and fast $(40 \mathrm{~Hz})$ oscillations. Ketamine and xylazine anesthesia. Simultaneous recording from five leads (1-5) in the depth (0.8-1 mm) of areas 7 and 5 in the suprasylvian gyrus (see cortex figurine at bottom). EEG activation was induced by a pulse train $(300 \mathrm{~Hz}, 2 \mathrm{sec})$ to the PPT nucleus. Four windows, of $12 \mathrm{sec}$ each, depict cross-correlations (CROSS) between various leads (left column) and FFTs derived from autocorrelation functions (right column) during the Control period before PPT stimulation, the Activation period (during and 10 sec after the pulse train), Post-activation period (before the slow oscillation recovered its full synchronization), and Recovery period. FFTs peaks of frequencies below $1 \mathrm{~Hz}$ are cut to maintain the same scaling for all panels and to reveal the presence of faster oscillations. During the control period, cross-correlations between close electrodes have peaks of 0.9 , whereas the peak drops to 0.7 between more distant sites $(4-5)$. All time lags are $\sim 0$ msec. During the activated epoch, the fast oscillations were between 35 and $40 \mathrm{~Hz}$ (see FFT), and cross-correlations indicate synchronization of rhythms around $40 \mathrm{~Hz}$, with time lags of $\sim 0 \mathrm{msec}$. Note marked difference between the peaks of correlations among neighboring electrodes (0.6) and distant electrodes (0.2). During the Post-activation period, the slow oscillation reappears yet is not fully synchronized; time lags between various leads are unaligned, indicating that the synchronization of neuronal pools is achieved gradually. Recovery is identical to Control. 


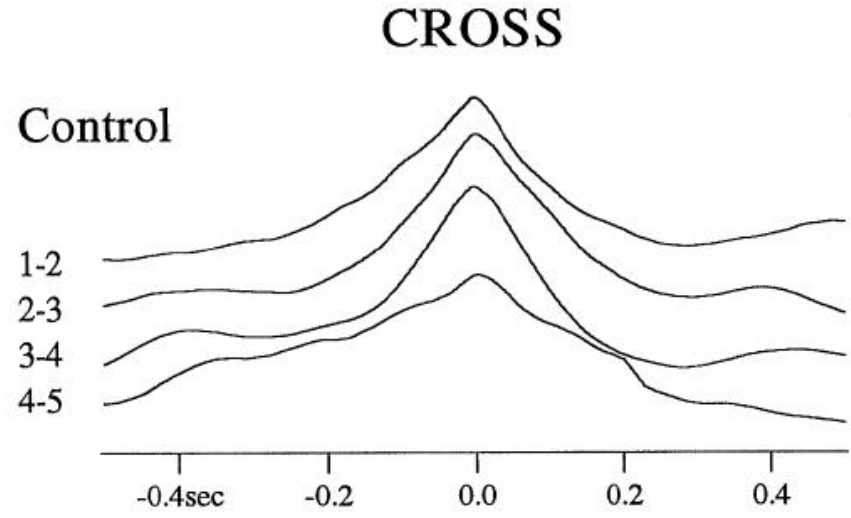

Activation
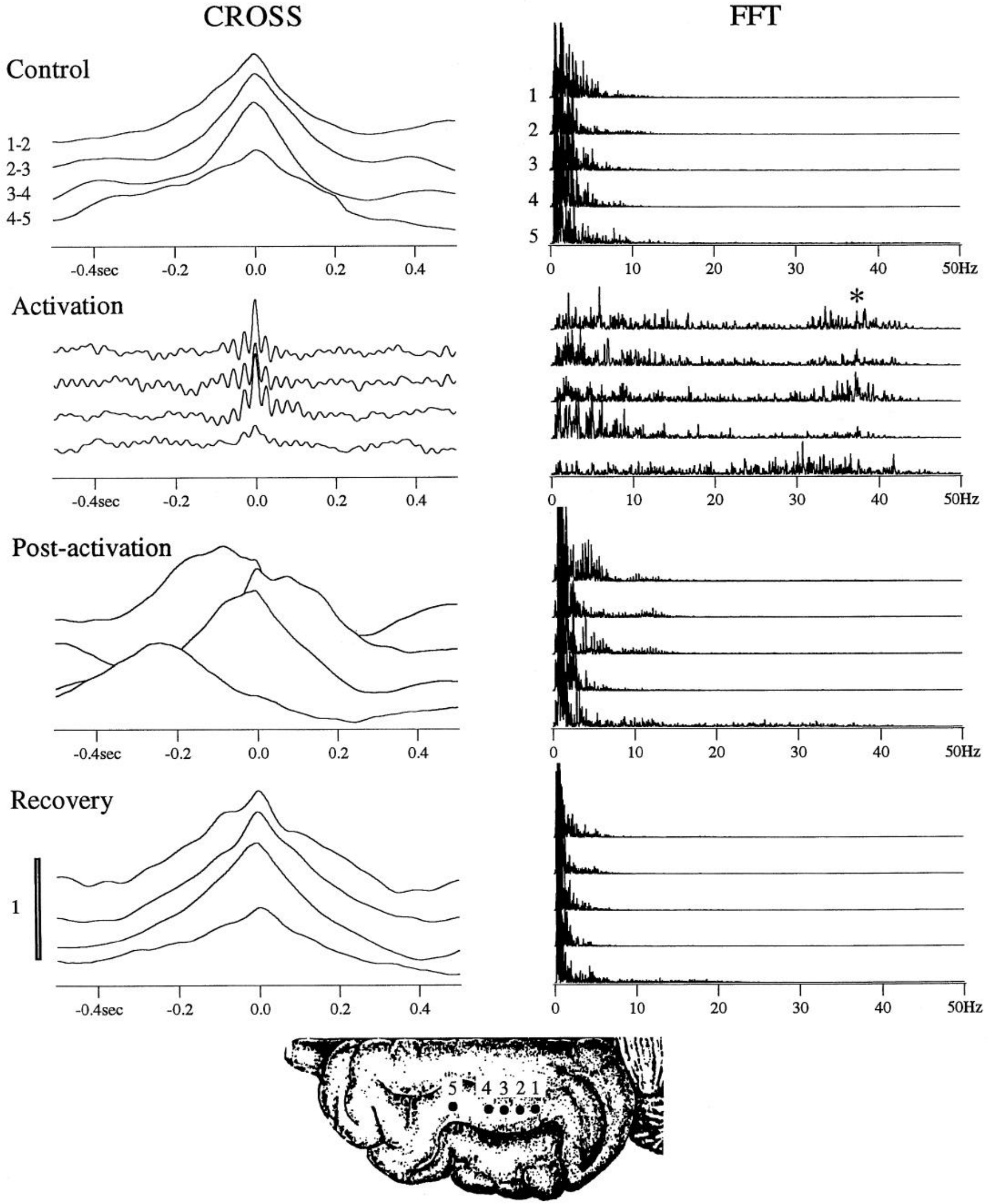
fast rhythms during the PPT-activated period [the cross-correlations indicating a clear-cut synchronization of the $40 \mathrm{~Hz}$ rhythm among adjacent cortical foci (1-2 mm apart) but reduced synchronization between more distant foci (electrodes 4 and 5 in Fig. 12)]; (3) disappearance of fast rhythms at 15-20 sec after the PPT pulse train, a period when the slow oscillation reappeared without, however, recovering its full synchronization between several cortical leads; and (4) recovery of the control pattern $25 \mathrm{sec}$ after the brainstem activating stimulation.

The extent of spatial synchronization of cortical fast rhythms during sleep-like epochs, when this activity was periodically interrupted during the prolonged hyperpolarizations of neurons, was not enhanced during activated epochs elicited by brainstem core stimulation, when the fast activity occurred in a sustained manner (see Figs. 1, 2). These statements are supported by Figure 13, which shows that the $30 \mathrm{~Hz}$ oscillation was synchronized within area 6 , but not among activities recorded from areas 6 and 5, 10 $\mathrm{mm}$ apart (see also below, Fig. 16, from behaving cat), and that the absence of coherence between distant areas was not changed after a pulse train to the mesopontine LDT nucleus that succeeded in producing clear signs of EEG activation.

\section{Intracortical synchronization of fast rhythms during sleep and arousal in behaving animals}

We conducted experiments on sleep and wake states in unanesthetized cats to determine to what extent the EEG cellular relationships during the slow oscillation under ketamine and xylazinc anesthesia are valid during natural sleep rhythms and whether the brainstem-elicited coherent fast cortical rhythms are similar to those occurring upon behavioral arousal, and if so, whether the patterns of intracortical synchrony of fast rhythms observed during activated epochs in acute experiments can also be observed during natural states of vigilance. We were surprised by the striking similarity between the paltern of slow uscillation under anesthesia and during natural sleep as well as by the relations between the fast rhythms $(20-40 \mathrm{~Hz})$ and different components of sleep-wake activity patterns that proved to be basically identical to those resulting from acute experiments.

During resting sleep, field potentials simultaneously recorded from the depth of several (somatosensory, motor, and suprasylvian association) areas showed the prevalence of a slow oscillation, between 0.7 and $0.9 \mathrm{~Hz}$, very similar to the slow oscillation recorded under ketamine and xylazine anesthesia. Nonethcless, some features differentiate anesthesia from sleep patterns. Under anesthesia, the slow oscillation is stereotyped, and this archetypal component of cortical activity groups the other two sleep rhythms, spindles at 7-14 Hz and delta at $1-4 \mathrm{~Hz}$ (Steriade et al., 1993e). During natural sleep, the slow oscillation is observed with the same components and temporal sequences as during anesthesia, but spindles and delta rhythms dominate the EEG activity in some epochs. The more complex patterns during natural sleep are seen in the field potential recordings from cortical areas 4 and 3 in Figure 14. The main component of the slow oscillation was a prolonged depth-positive wave during which neuronal firing was silenced (area 5 in Fig. 14), as was the case during the positive component of the slow oscillation under ketamine and xylazine anesthesia (see Figs. 5, 7, 8). The cellular discharges reappeared in close time relation with the sharp depth-negative deflection that follows the dcpth-positive EEG wavc. Arousal from sleep, associated with increased muscular tone and eye movements, replaced the prevalent low-frequency rhythms by fast EEG activity and tonically increased cellular firing (Fig. 14). Again, similar to the anesthesia pattern, fast rhythms $(20-25 \mathrm{~Hz})$ of local field potentials were diminished up to suppression during the depthpositive wave (see inset with filtered fast activity from area 5 in Fig. 14).

Cross-correlations and spectral analyses of cortical activities during transitions from sleep to wakefulness showed the replacement of low-frequency oscillations, prevalent during sleep, by fast rhythms ( $40 \mathrm{~Hz}$ in Fig. 15), as well as the synchronization of fast oscillations recorded from closely spaced $(1-2 \mathrm{~mm})$ cortical electrodes. Long periods of uninterrupted wakefulness, continuously associated with synchronized $40 \mathrm{~Hz}$ rhythms (as in Fig. 15), were not common. In many instances, such epochs with coherent fast oscillations were fugacious, and in spite of the fact that all electrographic and behavioral signs indicated wakefulness, synchronization was disrupted and reappeared for short epochs, without any detectable reasons.

The spatial extent of the synchrony between cortical foci exhibiting fast oscillations in chronic experiments was generally limited. We computed sequential cross-correlations during repeated transitions from resting sleep to wakefulness and extracted the dynamic correlation profile between cortical activities related to these shifts in the behavioral state. In this way, we obtained an evolutive view of the correlation strength and time lags between waves from closely located or more distant cortical areas $(n=7)$. The example in Figure 16 shows simultaneous recordings from four cortical sites, two with field potentials from precruciate area 4 and coronal area $3 \mathrm{~b}$, separated by $>5 \mathrm{~mm}$, and two with unit discharges and local field potentials from foci separated by $1.5 \mathrm{~mm}$ in the anterior suprasylvian area 5. The transitions from sleep to short arousals or a more sustained epoch of waking are shown by EEG activation, increased muscular tone, and during the longer period of wakefulness, tonic firing of neurons. The cross-correlations between fast rhythms recorded from four cortical foci did not change throughout the $50 \mathrm{sec}$ period including three transitions from sleep to brain arousal (Fig. 16). Also, similar to data from acute experiments (see Fig. 13), closely located foci in area 5 displayed strong coherence, with consistent time lags between them (generally 1-3 msec) during both sleep and waking, whereas the time lags between fast rhythms recorded from the more distant foci in areas 4 and 3 did not show a consistent pattern, being dispersed at long intervals (Fig. 16).

The transition to REM sleep is associated with EEG activation, muscular atonia, and eye movements (Fig. 17A). Although the neuronal firing was suppressed during the prolonged depthpositive waves of slow sleep oscillation, cortical cells became tonically active during the transition from slow-wave slecp to REM sleep, in advance of overt EEG activation, and they increased their discharge frequencies in close time relation with PGO waves (Fig. 17A). We found in-phase synchronization between fast $(20-40 \mathrm{~Hz})$ waves recorded from the cortical surface and depth in simultaneously recorded neocortical areas (Fig. $17 B$ ), as was found for the events described during wakefulness. Similar to the spatially limited intracortical coherence observed in the wake state, the synchronization of fast rhythms was restricted to closely located cortical sites. Occasionally, coherent fast rhythms were seen over larger distances, up to $10-12 \mathrm{~mm}$; in those instances, however, the correlation peak was high (above $0.7-0.8$ ) between surface and depth activities within the same column in areas 17 or 18 , whereas it dropped to $0.2-0.3$ when those areas were correlated (Fig. 17B). 

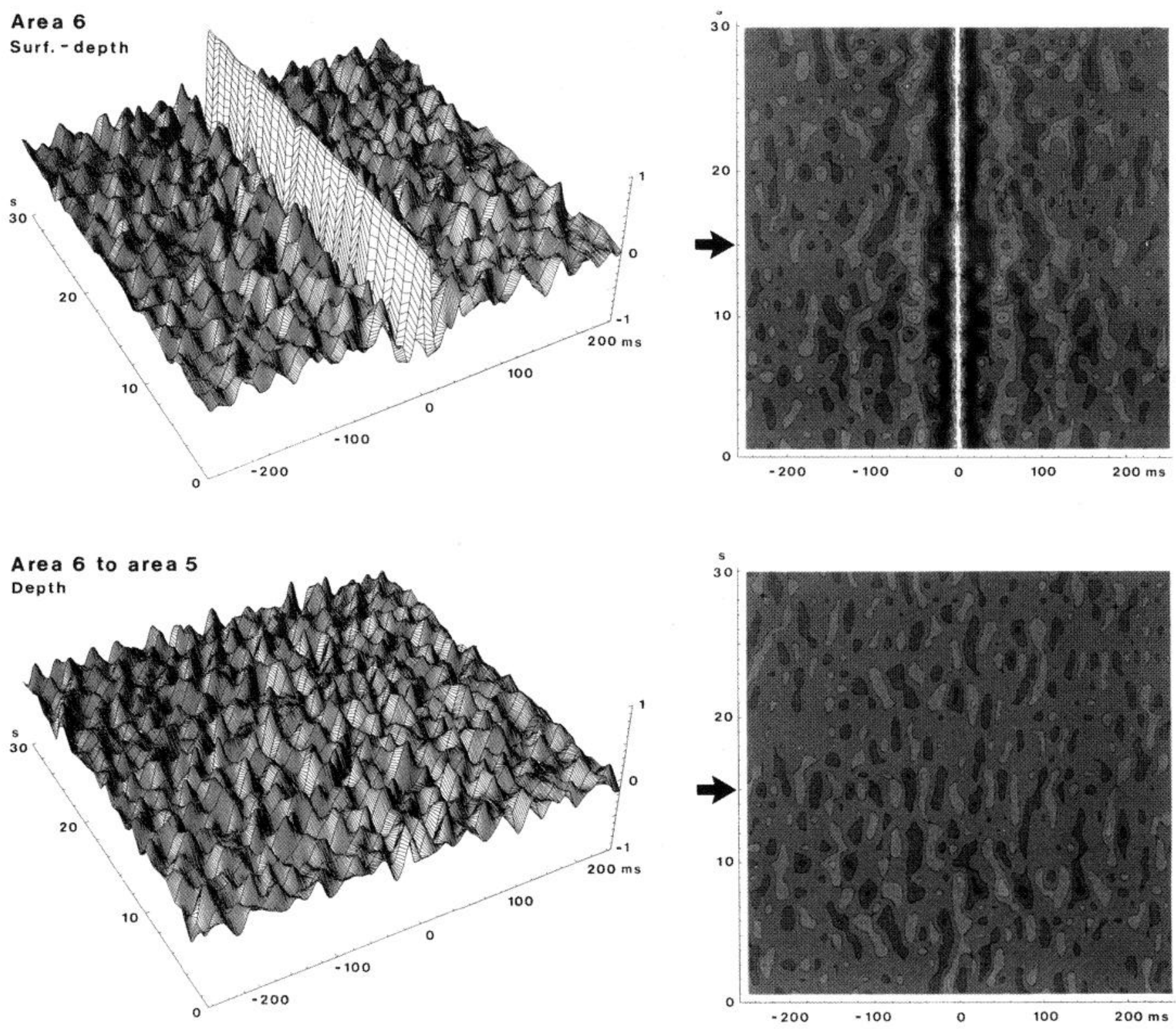

Figure 13. Sequential cross-correlations between intracolumnar (Surf-depth in Area 6) and more distant foci (Area 6 to area 5). Ketamine and xylazine anesthesia. The field potentials from the two cortical areas were recorded with multiple arrays of electrodes (as in Fig. 2, same animal). An epoch of 30 sec, with waves digitally filtered between 15 and $80 \mathrm{~Hz}$, was sliced in 30 consecutive windows of 1 sec each, and sequential cross-correlations (see Data analysis in Materials and Methods) were performed. Top panels demonstrate highly coherent, in-phase activities between the surface and depth (1 mm) in the same column. Note in the contour map the presence of aligned secondary peaks (bordering the dark valleys adjacent to the central white peaks) as evidence of constant oscillations around $25 \mathrm{~Hz}$ throughout the investigated epoch. Bottom panels display the lack of coherent fast rhythms between area 6 and area 5. In both top and bottom, arrows indicate the application of a pulse train $(300 \mathrm{~Hz}, 1 \mathrm{sec})$ to the mesopontine LDT nucleus. Although the slow oscillation was abolished (data not shown) (see Figs. 1-2), there was no potentiating effect on the coherence between distant cortical sites (areas 6 and 5).

\section{DISCUSSION}

The present experiments demonstrate that fast cortical rhythms $(20-40 \mathrm{~Hz})$ are part of background electrical activity in all states of vigilance, without necessarily being triggered by optimal sensory stimuli, and that they are potentiated during states of brain activation. The robust coherency of fast spontaneous rhythms, albeit their synchronization is spatially more restricted than that of slow oscillations, challenges the conventional idea that brain-activated states are associated with a complete desynchronization of cortical rhythms.

\section{In-phase, surface-to-depth fast cortical rhythms and their spatially limited horizontal coherence}

The polarities of the two major components building up the slow sleep oscillation $(<1 \mathrm{~Hz})$, i.e., the prolonged depth-positive wave and the subsequent negative wave, reversed in all cases, mainly from 0.25 to $0.4 \mathrm{~mm}$. However, in an overwhelming majority (90\%) of the same tracks through a variety of areas, the fast rhythms were in phase from the surface to the deepest cortical layers (Figs. 1, 3), whereas their amplitudes were greatly reduced in the underlying white matter (Fig. 


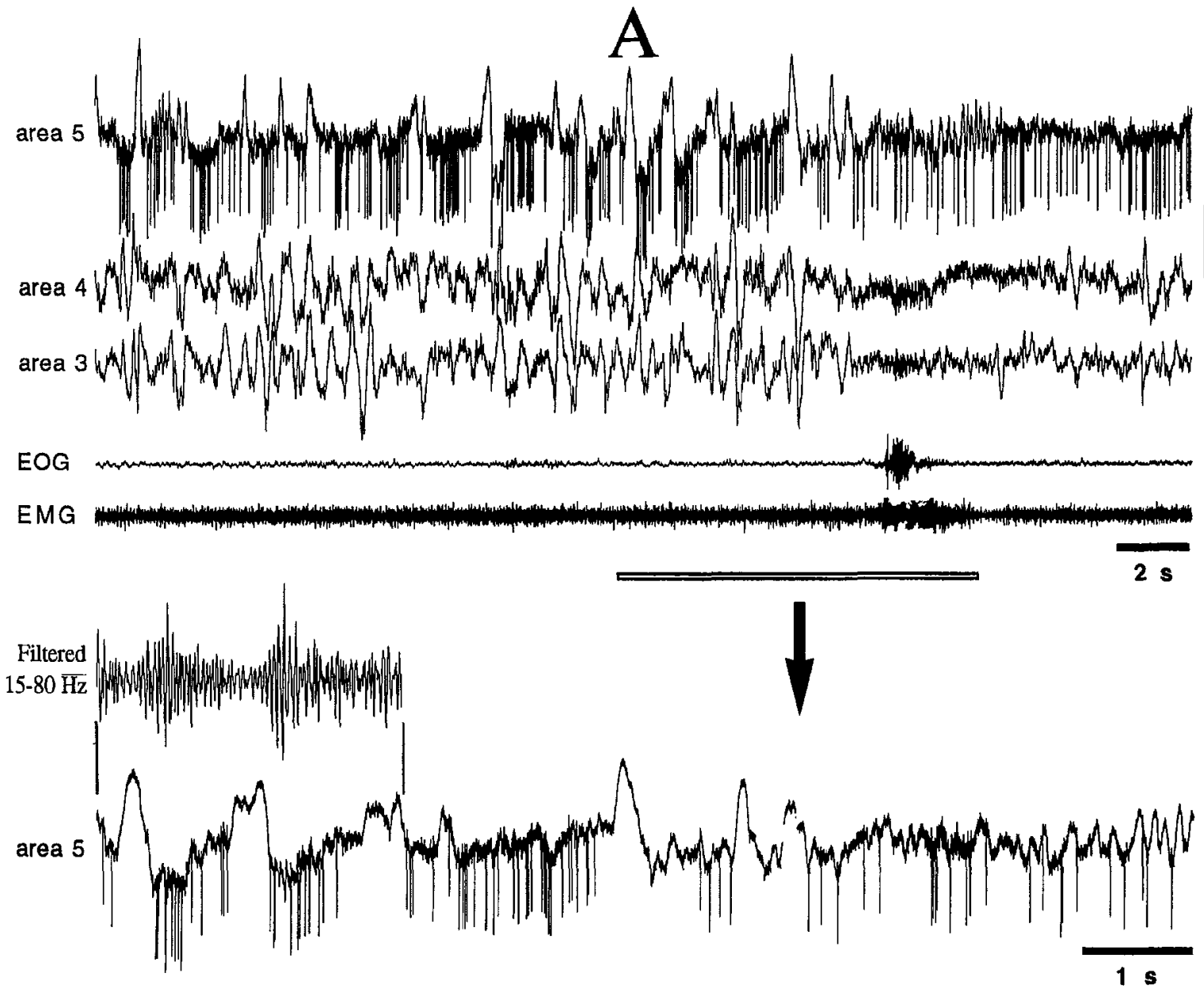

B
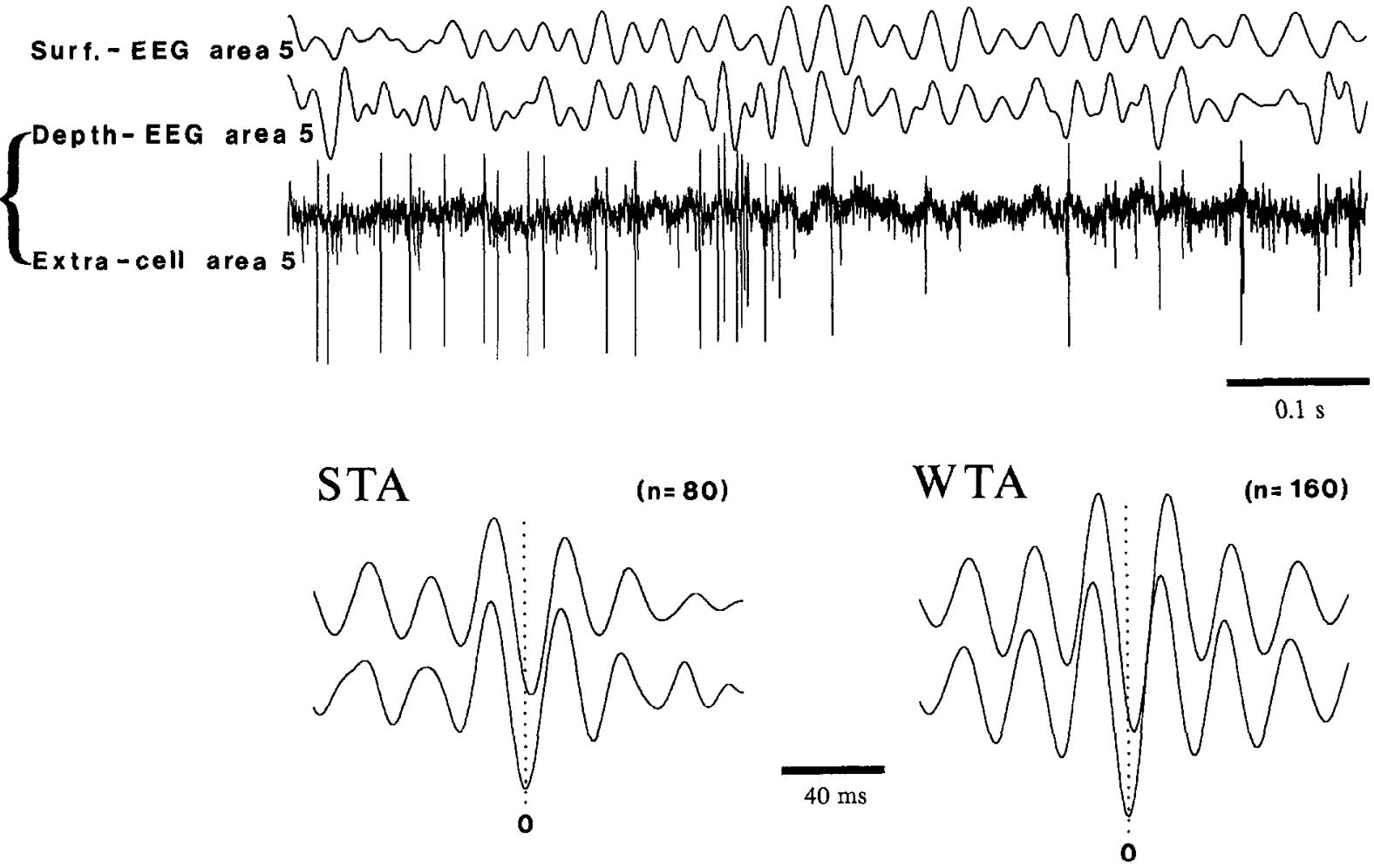

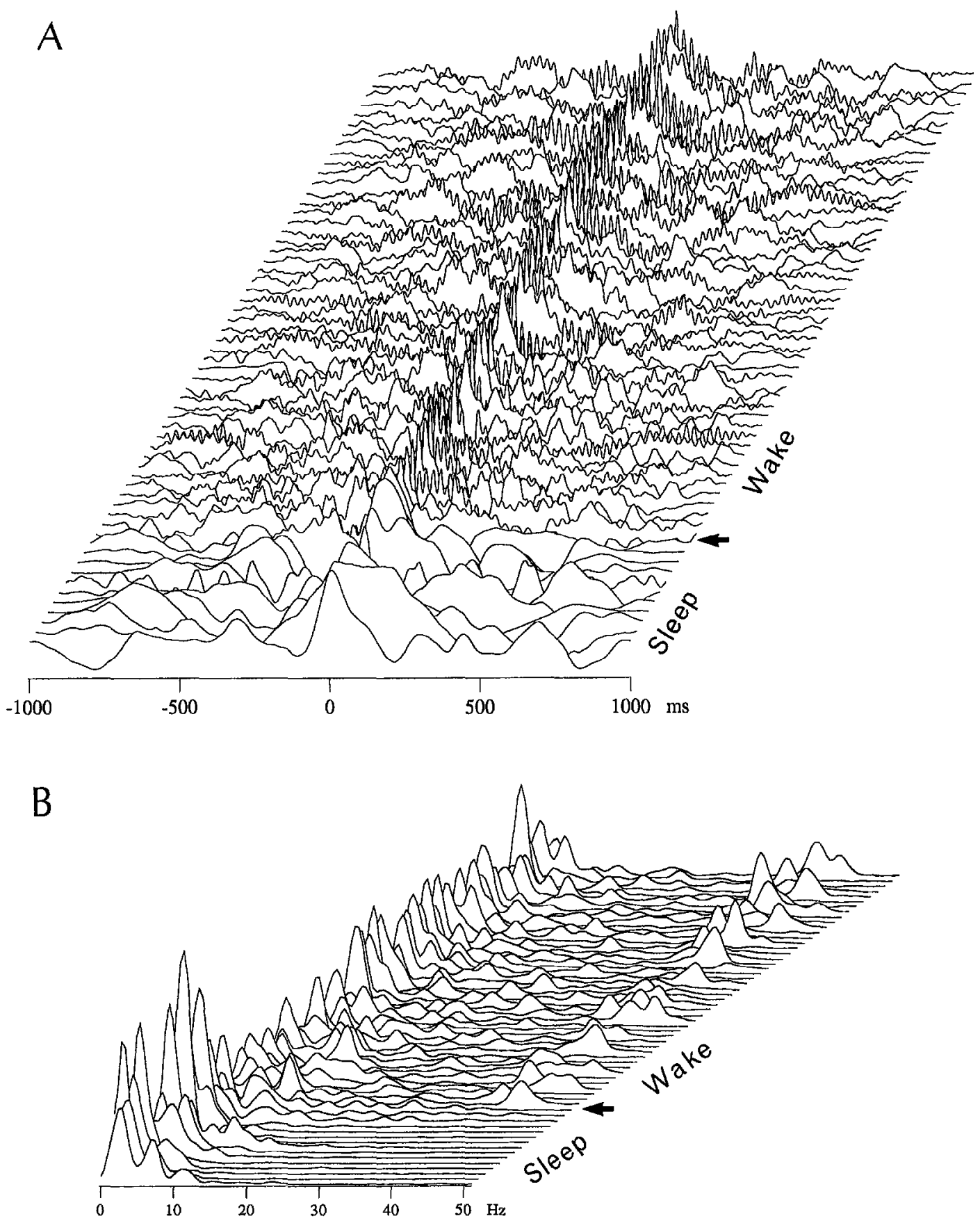

Figure 15. Coherent fast spontaneous thythms $(\sim 40 \mathrm{~Hz})$ in close cortical foci upon arousal from sleep in chronically implanted animal. $A$, Cross-correlations from $1 \mathrm{sec}$ sequential windows between field potentials recorded from close $(1 \mathrm{~mm})$ sites in area 5 . During the first sweeps, slow sleep oscillation dominates the correlations, and their peaks are aligned around time 0 . Upon awakening (arrow), cross-correlations become dominated by in-phase fast frequencies. $B$, The cross-spectrum shows the predominance of low frequencies $(<10 \mathrm{~Hz})$ during sleep and the appearance of fast activities (around $40 \mathrm{~Hz}$ ) after arousal. Cross-correlation functions are insensitive to variation in amplitudes of waves in original data, and therefore the decreased amplitudes of low-frequency oscillations upon arousal are less evident in cross-spectra.

Figure 14. Fast rhythms (20-25 Hz) during depth-negative cortical EEG waves in natural sleep, their suppression during depth-positive EEG waves, and in-phase (surface-to-depth) fast oscillations during natural waking in behaving animals. $A$, Simultaneous recording from cortical area 5 (field potentials and unit discharges) and field potentials from depths of areas 4 and 3, together with EOG and EMG. Arousal from sleep is indicated by EEG activation, eye movements, and increased muscular tone. Part marked by horizontal bar is expanded in the bottom panel (arrow) depicting only activitics from area 5; left part (between lines) is further depicted above, with increased amplitudes and filtered activities (between $15 \mathrm{and} 80 \mathrm{~Hz}$ ). Note $20-25 \mathrm{~Hz}$ waves during depth-negative components of the slow oscillation and diminution up to disappearance of fast waves during depth positivities. Also note the striking similarities between the patterns of slow sleep oscillation $(0.9 \mathrm{~Hz})$ in chronically implanted, unanesthetized, naturally sleeping animals and the same oscillatory type in animals under ketamine and xylazine anesthesia (see Fig. 1 and other similar figures). $B$, Another chronically implanted cat, showing in-phase-, surface-, and depth-recorded fast field potentials at $\sim 40 \mathrm{~Hz}$. Spike-triggered averages (STA) of surface- and depth-EEG waves, with action potentials of area cell 5 at time 0 (see above recordings of neuronal firing together with surface- and depth-EEG). Wave-triggered averages (WTA) with peak negativity of depth field potentials at time 0 . Note short time lags $(2-3 \mathrm{msec})$ between depth- and surface-EEG fast oscillation. 

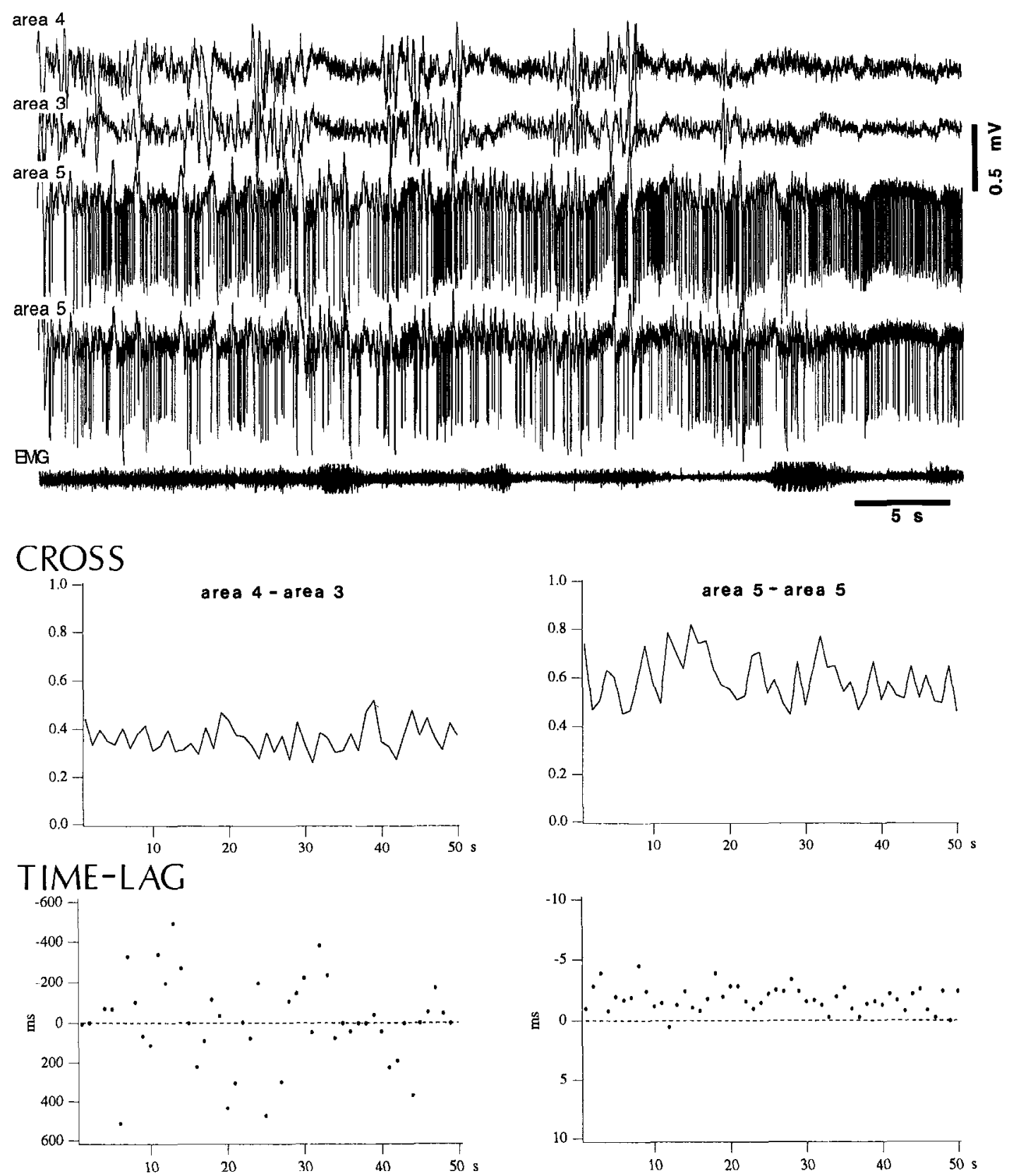

Figure 16. Dynamic correlation profile (DCP) between cortical activities recorded from close (two foci in area $5,<1 \mathrm{~mm}$ apart) and more distant sites (areas 4 and 3). Alternating states of sleep and arousal in behaving animal. Top panel depicts simultaneous recordings of field potentials in areas 4 and 3 , field potential and unit discharges (through the same microelectrodes) from two close sites in area 5 and EMG. Bottom panels illustrate DCP derived from sequential cross-correlations (activities filtered between $15 \mathrm{and} 80 \mathrm{~Hz}$ ). Cross-correlations were computed in sequential windows of 1 sec during the sleep and waking episodes displayed in the top panel. For each correlation, the highest peak was detected and its value and latency, respectively, were plotted in the upper (CROSS) and lower (TIME-LAG) graphs. They represent a contracted equivalent of the sequential field correlation and of the ensuing three-dimensional surfaces, as shown in Figure 13. The two plots (CROSS and TIME-LAG) have to be read together because their information is complementary. The correlations between areas 4 and 3 show low values $(\sim 0.4)$ combined with sparse time lags, often far beyond the period of fast activities. Conversely, the synchrony between the two closely located foci in area 5 consisted of higher correlation values $(0.6-0.8)$ and short time lags $(<4 \mathrm{msec})$.

4). The fact that depth-negative fast wavelets from both upper and deeper layers were associated with action potentials of neurons recorded as superficially as $0.15-0.2 \mathrm{~mm}$ as well as to discharges of cells in deep cortical layers (Fig. 5) indicates that fast oscillations are generated across the cortical depth and substantiate the surprising finding of their absence of reversal. Additional arguments against the possibility of volume-conducted fast oscillations are short time lags
(2-3 msec) between surface and depth activities (Figs. 5, 14) and the fact that the synchronization of fast waves is spatially limited. The in-phase, surface-to-depth coherency of fast waves was established on the basis of visual inspection of long periods of activity combined with averaged cross-correlations and spike-triggered averages. However, very short epochs displayed changing patterns with slightly different frequencies in various cortical layers, leading to prevalent 

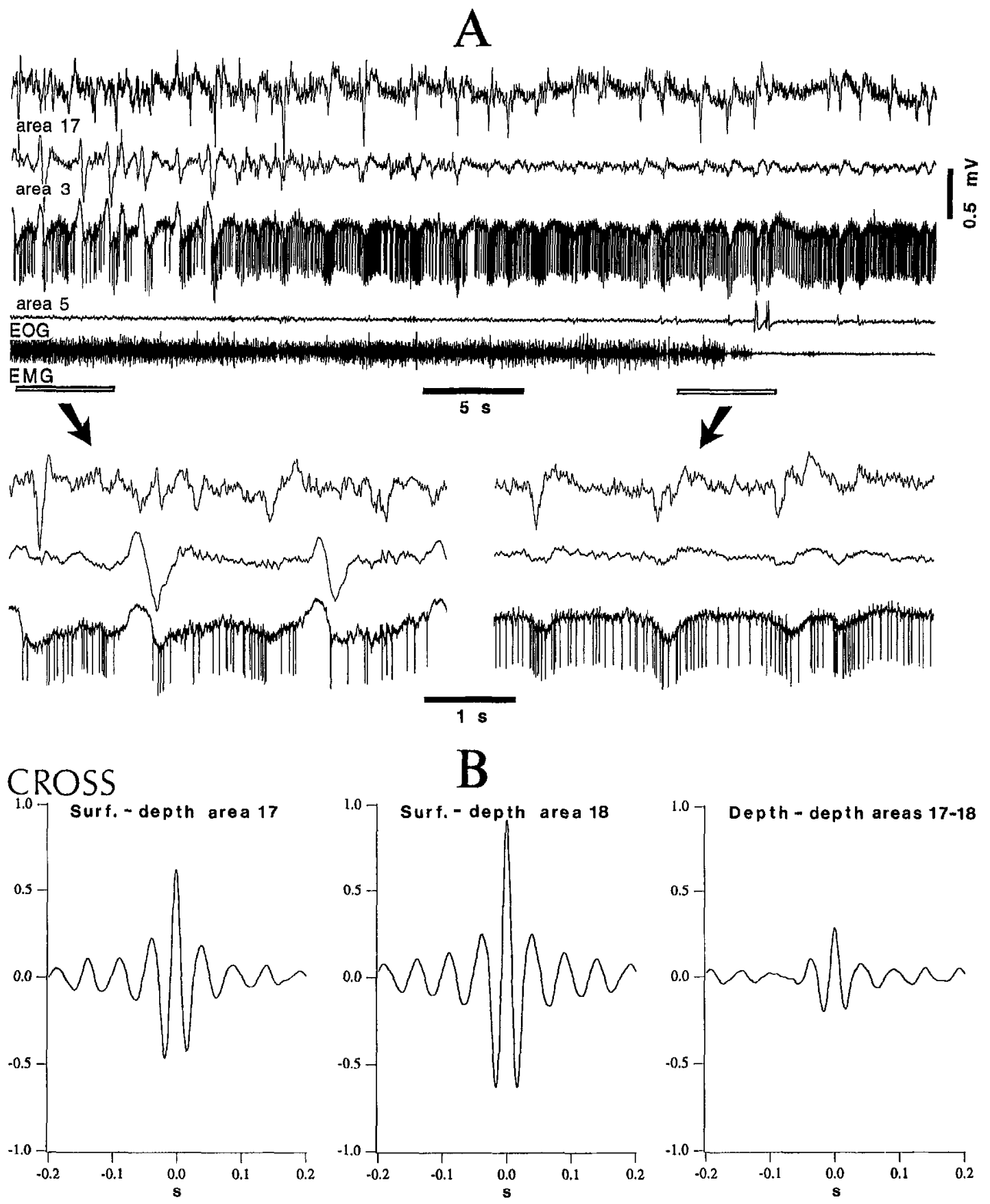

Figure 17. Transition from slow-wave sleep to REM sleep in chronically implanted animal. $A$, Simultaneous recordings of field potentials from the depth of areas 17 and 3, and single unit and field potentials from area $5, E O G$, and $E M G$. Two epochs during slow-wave sleep and REM sleep, indicated by horizontal bars, are expanded below (arrows) to depict changing activities in areas 17, 3, and 5. Note suppressed neuronal firing (area 5) during the depth-positive FFG wave during slow-wave sleep (left) and depth-negative PGO potentials associated with increased cell firing during REM sleep (right). $B$, Another animal during REM sleep. Cross-correlations (CROSS) between surface- and depth-recorded fast activities $(\sim 20 \mathrm{~Hz})$ in area 17 and rostral part of area 18 , as well as between depth activities in areas 17 and 18.

phase opposition between the surface and a circumscribed depth of cortex, at $0.8-1 \mathrm{~mm}$ (see Fig. 6), in $10 \%$ of the recordings. In sum, the depth profile of fast neocortical oscillation is a largely prevalent in-phase activity, with exceptions during brief epochs when changing frequencies of the rhythm are observed within different cortical layers.

The absence of depth reversal of the fast oscillation suggests that the synchronous synaptic inputs and intrinsic currents, conjointly generating such fields, are uniformly distributed along the vertically organized dendritic core conductors. Thus, the current flow would be mainly attributable to transmembrane components and less so to internal longitudinal components. This interpretation is supported by the present experiments showing neuronal spike trains superimposed on negative field potentials throughout the cortical depth (see Fig. 5). Vertically distributed currents cannot be ruled out, however, inasmuch as current-source-density analyses show alternatively distributed microsinks and microsources (Steriade and Amzica, 1996). The lower intensity of these 
vertical currents, compared with that of transmembrane currents, may explain the absence of potential reversal.

During brain-activated epochs, myriads of thalamic and cortical inputs excite the neurons throughout the cortex, from the most superficial to the deepest layers. Cortical-projecting thalamic nuclei are implicated in the genesis of fast spontancous oscillations $(20-40 \mathrm{~Hz})$ and in their transfer to cortex, as demonstrated by voltage (depolarization)-dependent or synaptically elicited $40 \mathrm{~Hz}$ rhythms in thalamocortical neurons (Steriade et al., 1991b, 1993b; Ghose and Freeman, 1992) and by brainstem-induced fast cortical oscillations that are mediated by the thalamus as they survive total lesions of the basal forebrain (Steriade et al., 1991b). In addition to their prevalent projections to midlayers, many thalamocortical axons innervate layers I and VI (Steriade et al., 1990b). The superficial cortical projections characterize in particular rostral intralaminar neurons (Jones, 1975; Steriade et al., 1984; Cunningham and LeVay, 1986), which represent an important input source for pericruciate, suprasylvian, and marginal areas explored in the present study. As for corticocortical pathways, long-range intrahemispheric horizontal projections, linking neuronal pools having widely separated receptive fields, are present in most sensory and motor fields (Gilbert, 1992; Aroniadou and Keller, 1993; Cauller and Connors, 1994; Scannell et al., 1995). These connectional patterns via long-distance intracortical horizontal pathways, which primarily involve dendrites in layers II/III and are followed by activation processes through vertical intracolumnar projections, seem to be basically similar in many, functionally different cortical fields. The assumption that fast cortical oscillations are mainly attributable to synaptic events involving the dendrites of pyramidal cells, as major targets of corticocortical (and thalamocortical) projections, is corroborated by ampler oscillations at $35-40 \mathrm{~Hz}$ in presumed dendritic recordings (see Fig. 9). In addition to the recurrent EPSPs produced by collaterals of pyramidal neurons, mediated by both NMDA and non-NMDA receptors (Thomson et al., 1989; Salt et al., 1995), a substantial amount of excitation within intracortical projections also may result from disinhibition (Kisvárday et al., 1993).

The coherence of fast rhythms decreased with distance and became weak over $5 \mathrm{~mm}$. The short-range synchronization of fast rhythms observed in the present study is at variance with the intracortical synchronization of slow sleep rhythms (Amzica and Steriade, 1995a). Thus, the faster the oscillation, the shorter the distance of maximal coherence. Bullock and McClune (1989) measured the coherence in brains of rats and rabbits and similarly found that the coherence falls with the frequency of EEG waves and the distance between recorded leads. Also, in the experiments of Murthy and Fetz (1992) on behaving monkeys, the proportion of coherent oscillations recorded from two different cortical foci decreased with the distance between these sites.

\section{Intrinsic properties, network activity, and modulatory systems underlying coherent fast rhythms}

The fact that fast rhythms were suppressed during the long-lasting hyperpolarizing phases of slow sleep oscillation, that they were elicited by depolarizing current pulses, and that their amplitudes were enhanced upon brainstem-elicited or natural arousal simultaneously with an increase in synaptic activities suggests that a constellation of factors should be considered when discussing the generation of this activity.

The role of intrinsic properties of cortical neurons in the production of fast oscillations was demonstrated in neurons from slices of frontal cortex (Llinás et al., 1991; Gutfreund et al., 1995) as well as in corticothalamic and corticocortical cells recorded in vivo (Nuñez et al., 1992). We showed (Fig. 10) that after depolarizing current pulses, high-frequency spike bursts recur at fast frequencies $(30-40 \mathrm{~Hz})$, reminiscent of the patterns displayed by fast-conducting thalamocortical intralaminar neurons (Steriade et al., 1993b). Synaptic activities may boost the intrinsic properties of cortical neurons. In our experiments, depolarizing current pulses elicited action potentials grouped within sequences at $30-40 \mathrm{~Hz}$ that were potentiated by background synaptic activities, whereas pulses with the same parameters applied during hyperpolarization of cells revealed only the slowly adapting pattern of regular spiking neurons (Fig. 11). This suggests that fast oscillations occur as a result of combined intrinsic cellular properties and synaptic activities. The prevalent role of rhythmic synaptic activities in triggering the visually evoked fast oscillations of cortical neurons was also emphasized in whole-cell patch recordings from area 17 , in vivo (Jagadeesh et al., 1992). A similar conclusion was drawn by Buzsáki and his group (Bragin et al., 1995; Ylinen et al., 1995), who showed that the intracellularly recorded fast field oscillation $(200 \mathrm{~Hz})$ in the $\mathrm{C} \wedge 1$ hippocampal region is not exclusively dependent on membrane currents intrinsic to pyramidal cells, but is a network-driven phenomenon with critical participation of putative local inhibitory cells.

In addition to the synaptic activities imposed by local cortical networks, generalized modulatory systems exert decisive actions in promoting fast oscillations. Because these rhythms are present during both waking and REM sleep (Llinás and Ribary, 1993; present data) and because monoamine-containing neurons are virtually silent in REM sleep (Steriade and McCarley, 1990), we focus on cholinergic and glutamatergic activating systems that are active in both waking and REM sleep. Brainstem-thalamic neurons recorded from mesopontine cholinergic nuclei increase their firing rates and excitability with transition from resting sleep to either waking or REM sleep (Steriade et al., 1990a), two behavioral states with similar electrophysiological features in thalamocortical systems (Steriade et al., 1993c). In keeping with these results, the release of acetylcholine (ACh) in the thalamus is similarly enhanced during waking and REM sleep, compared with slow-wave sleep (Williams et al., 1994). In turn, thalamocortical cells, using glutamate as neurotransmitter, also display similarly enhanced discharge frequencies and antidromic as well as synaptic excitability in both waking and REM sleep (Glenn and Steriade, 1982). Thus the fast cortical oscillations $(20-40 \mathrm{~Hz})$ that outlast the stimulation of mesopontine cholinergic nuclei and are sensitive to cholinergic blockers are likely transmitted through the thalamus, because this effect survives total lesions of basal forebrain neurons (Steriade et al., 1991b). This bisynaptic, cholinergic-glutamatergic, brainstem-thalamic-cortical link acts concomitantly with the direct cholinergic-mediated fast cortical oscillation $(20-40 \mathrm{~Hz})$ in response to stimulation of nucleus basalis (Metherate et al., 1992). The excitatory input sources for driving basalis neurons are the glutamatergic projections from the upper brainstem core (Rasmusson et al., 1994). The production of fast oscillations through either brainstem-thalamic-cortical (cholinergic-glutamatergic) or brainstem-basalis-cortical (glutamatergic-cholinergic) circuits is primarily attributable to the depolarization of cortical neurons. The muscarinic actions of $\mathrm{ACh}$ consist of voltage-dependent membrane depolarization associated with increased input resistance, which is attributable to the decreased $\mathrm{K}^{+}$conductances (Krnjević et al., 1971; McCormick and Prince, 1986). Similar effects are likely exerted by thalamocortical neurons, because the activation of metabotropic glutamate recep- 
tors in cortical neurons results in prolonged depolarization, with a shift from bursting to tonic firing (Wang and McCormick, 1993).

Our results also demonstrate that far from exclusively characterizing the brain-active states of waking and REM sleep, fast oscillations are also present during the behavioral state of resting sleep or under general anesthesia with ketamine and xylazine. In these so-called "slow-wave" states, fast rhythms $(20-40 \mathrm{~Hz})$ appear during the prolonged depolarizing phases of the slow oscillation $(<1 \mathrm{~Hz})$ in cortical cells and are selectively suppressed during the long-lasting hyperpolarizations. The similar cellular EEG relations during anesthesia and behavioral sleep validate, in the natural condition of chronically implanted naturally sleeping animals, previous data on the intracellular correlates of slow EEG oscillations in cortical, thalamic reticular, and thalamocortical neurons obtained under ketamine and xylazine anesthesia (Contreras and Steriade, 1995).

\section{Fixed or variable frequencies of fast oscillations?}

The widely used term " $40 \mathrm{~Hz}$ " rhythm would imply a fixed, narrow, or preferred frequency. However, cellular studies have revealed that a majority of neocortical neurons display a broad frequency range (from 10 to $30-40 \mathrm{~Hz}$ ) of subthreshold responses to depolarizing current pulses, whereas only a minority of cells proved to be narrow frequency oscillators in the range of $35-50 \mathrm{~Hz}$ (Llinás et al., 1991) or at fixed frequencies $\sim 30 \mathrm{~Hz}$ (Nuñez et al,, 1992).

After we examined our data, it became clear that the frequency of fast oscillation could change in the same intracellularly recorded neuron or in the same local field potential recording (reflecting summated PSPs and intrinsic currents in a pool of neurons) without any visible alterations in the global state of vigilance, as monitored by EEG activity and behavioral signs in chronically implanted animals. The fluctuations in frequencies occurred within epochs $<5 \mathrm{sec}$ or even $<1 \mathrm{sec}$ (see such changes in frequency in the detail of Fig. 1, during the activated state). In fact, the frequency of fast oscillations, $>15 \mathrm{~Hz}$, increased with the progression of the depolarizing envelope of the slow oscillation (lasting for $<1 \mathrm{sec}$ ) to eventually reach the highest frequency $(35-40 \mathrm{~Hz})$ and the amplest membrane fluctuations toward the end of this phase (see the intracellular recording in Fig. $8 A$ and also the filtered extracellular activities in Fig. $5 B$ ). The fast oscillations during brainstem-elicited activated epochs or natural waking were at 35-40 Hz (Figs. 1-5) or higher frequencies, whereas in experimental conditions of sleep or arousal, the fast oscillation was at $25-30 \mathrm{~Hz}$ (see Fig. 14). Similar variations, with peak frequencies shifting by a factor of 2 in as few as $10 \mathrm{sec}$, were reported by Ghose and Freeman (1992) in cortical neurons driven by the same visual stimuli. It should be mentioned that one of the drawbacks of the spectral analysis is that it conceals the information about the temporal evolution of the signal. This is why one never knows by looking at a broad spectrum whether it is generated by an oscillation with continuously changing frequencies, by an oscillation with stable frequencies at multiple peaks, or by a combination of the two.

Do the variations in the frequency of fast rhythms reflect different functional states? Possibly, if by functional state we mean a microepoch in which a limited number of "spontaneous" PSPs are added to the network and succeed in altering the frequency of the oscillation. The answer to this question is negative if we refer to global functional states or to distributed phasic events. To say the least, it would be difficult to relate the changes in oscillatory frequencies from 20 to $40 \mathrm{~Hz}$ during microepochs with real operations in the performing brain.

Based on these data and considerations, we believe that there is no reason to split fast rhythms into two distinct categories, $\beta$ and $\gamma$, the former between approximately 15 and $30 \mathrm{~Hz}$ and the latter $>30 \mathrm{~Hz}$, as some clinical or experimental studies used to do. In fact, the Greek letters indicating different bands of EEG rhythms have no logic. Their alphabetical sequence neither follows a given progression in frequency nor reflects the behavioral correlates of oscillations; it can only be understood by considering the chronological order of description of these rhythms, which started in the late 1920s (Niedermeyer, 1993). This is why we simply call the oscillations $>20 \mathrm{~Hz}$ fast rhythms.

\section{Concluding remarks: synchronization of fast rhythms, brain activation, and conscious events}

This article provides experimental data indicating that whereas the synchronizing factors underlying low-frequency $(<15 \mathrm{~Hz})$ sleep rhythms are decoupled with transition to either waking or REM sleep, the amplitudes of fast neocortical rhythms (mainly $20-40 \mathrm{~Hz}$ ) are enhanced, although the horizontal extent of synchronized fast cortical oscillations in arousal is more limited than that of slow oscillations during sleep. Our data challenge the term "EEG-desynchronized states" applied to waking and REM sleep in most past and contemporary studies. Desynchronization should therefore be replaced by "activation," a term that was proposed by Moruzzi and Magoun (1949). The theoretical implication of this term is that fast spontaneous activities during arousal are associated with an increased neuronal responsiveness, including inhibition in the activated process (Jasper, 1958), thus providing both quick and finely tuned reactions (Steriade et al., 1990b; Steriade et al., in press).

Some postulate that the fast (so-called $40 \mathrm{~Hz}$ ) oscillations are implicated in consciousness and that sensory-induced fast oscillatory responses, occurring synchronously in spatially distributed neuronal clusters, would serve to bind the particular features of an object into a global percept (Crick and Koch, 1990; Llinás, 1990; Singer, 1990a). The alternative view considers that fast rhythms do not require optimal sensory stimuli and are not necessarily related to higher conscious processes but are part of the background activity of the brain (Steriade et al., 199.b), reflecting the depolarization of thalamic and cortical neuroris, a physiological condition that is potentiated by central core modulatory systems (Steriade, 1993). The present data show that coherent fast rhythms occur spontaneously, that they are present during the periodic depolarizing components of the slow oscillation in states associated with mental annihilation (such as slow-wave sleep or deep anesthesia), and that brain arousal increases the synchronized firing of cortical neurons during these oscillations. The fact that fast thythms also appear during resting sleep when thalamocortical gates are closed to external signals does not exclude the possibility that similar intrinsic cellular properties and network operations would underlie binding operations, significant for cognitive experiences, during brain open and adaptive states. In visual cortex, the amplitudes of fast oscillatory responses to photic stimuli are selectively enhanced, and the coherency of multiunit discharges is increased by setting into action brainstem ascending activating systems (Steriade, 1968; Steriade et al., 1968; Singer, 1990b). Such changes in electrical activities are also expected to occur in natural transitions from rest, inattentiveness, or drowsiness to arousal and highly motivated states, as is probably the case with the oscillations at $25-35 \mathrm{~Hz}$ that appear in monkeys when 
they retrieve raisins from unseen sites (Murthy and Fetz, 1992). Thus, states characterized by an increased level of vigilance, associated with depolarization of thalamic and cortical neurons (Steriade et al., 1990b, 1993c), favor the synchronization of fast oscillating neurons. In other brain regions it was shown that if excitatory inputs are coincident with the depolarizing phase of the subthreshold oscillation, they are likely to cause the neurons to fire (Lampl and Yarom, 1993), thus increasing the electroresponsiveness of cells and their impact on target structures.

We hypothesize that thalamic and cortical neurons that oscillate at fast frequencies $(20-40 \mathrm{~Hz})$ during states associated with their depolarization, but the synchrony of which is very weak or spatially limited or both, would become robustly coherent for short periods of time after a relevant stimulus in the wake state or an internally generated drive in REM sleep. Our data (Fig. 3) showed that the PPT-evoked responses, resembling the PGO potentials of REM sleep, are followed by synchronization of fast cortical oscillations over a time window of $\sim 600 \mathrm{msec}$. Similar data may be obtained by using significant sensory stimuli during wakefulness. These aspects, together with the involvement of dorsal thalamic nuclei in the synchronization of fast oscillations in related neocortical territories, are now being investigated (in preparation).

\section{REFERENCES}

Amzica F, Steriade M (1995a) Short- and long-range neuronal synchronization of the slow $(<1 \mathrm{~Hz})$ cortical oscillation. J Neurophysiol 73:20-39.

Amzica F, Steriade M (1995b) Disconnection of intracortical synaptic linkages disrupts synchronization of a slow oscillation. $J$ Neurosci 15:4658-4677.

Aroniadou VA, Keller A (1993) The patterns and synaptic properties of horizontal intracortical connections in the rat motor cortex. J Neurophysiol 70;1553-1569.

Barlow IS (1993) The electroencephalogram: its patterns and origins. Cambridge: MIT.

Bendat JS, Piersol AG (1980) Engineering applications of correlation and spectral analysis. New York: Wiley.

Bouyer JJ, Montaron MF, Rougeul A (1981) Fast frontoparietal rhythms during combined focused attentive behaviour and immobility in cat: cortical and thalamic localization. Electroencephalogr Clin Neurophysiol 51:244-252.

Bragin A, Jandó G, Nádasdy Z, Hetke J, Wise K, Buzsáki G (1995) Gamma $(40-100 \mathrm{~Hz})$ oscillation in the hippocampus of the behaving rat. J Neurosci 15:47-60.

Bremer F, Stoupel N (1959) Facilitation et inhibition des potentiels évoqués corticaux dans l'éveil cérébral. Arch Int Physiol Biochim 67:240-275.

Bremer F, Stoupel N, Van Reeth PC (1960) Nouvelles recherches sur la facilitation et l'inhibition des potentiels évoqués corticaux dans l'éveil réticulaire. Arch Ital Biol 98:229-247.

Bullock TH, McClune MC (1989) Lateral coherence of the electrocorticogram: a new measure of brain synchrony. Electroencephalogr Clin Neurophysiol 73:479-498.

Cauller LJ, Connors BW (1994) Synaptic physiology of horizontal afferents in layer I in slices of rat SI cortex. J Neurosci 14:751-762.

Contreras D, Steriade M (1995) Cellular basis of EEG slow rhythms: a study of dynamic corticothalamic relationships. J Neurosci 15:604-622.

Crick F, Koch C (1990) Some reflections on visual awareness. Cold Spring Harb Symp Quant Biol 55:953-962.

Cunningham ET, LeVay S (1986) Laminar and synaptic organization of the projection from the thalamic nucleus centralis to primary visual cortex. J Comp Neurol 254:65-77.

Desmedt JE, Tomberg C (1994) Transient phase-locking of $40 \mathrm{~Hz}$ electrical oscillations in prefrontal and parietal human cortex reflects the process of conscious somatic perception. Neurosci Lett 168:126-129.

Dumont S, Dell P (1960) Facilitation réticulaire des mécanismes visuels corticaux. Electroencephalogr Clin Neurophysiol 12:769-796.

Eckhorn R, Bauer R, Jordan W, Brosch M, Kruse W, Munk M, Reitboeck HJ (1988) Coherent oscillations: a mechanism of feature linking in the visual cortex? Biol Cybern 60:121-130.
Franken P, Dijk DJ, Tobler I, Borbély AA (1994) High-frequency components of the rat electrocorticogram are modulated by the vigilance states. Neurosci Lett 167:89-92.

Freeman WJ, Van Dijk BW (1987) Spatial patterns of visual cortical fast EEG during conditioned reflex in a rhcsus monkey. Brain Rcs 422:267-276.

Frégnac Y, Bringuier V, Baranyi A (1994) Oscillatory neuronal activity in visual cortex: a critical re-evaluation. In: Temporal coding in the brain (Buzsáki G, Llinás R, Singer W, Berthoz A, Christen Y, eds), pp 81-102. Berlin: Springer.

Ghose GM, Freeman RD (1992) Oscillatory discharge in the visual system: does it have a functional role? J Neurophysiol 68:1558-1574.

Gilbert CD (1992) Horizontal integration and cortical dynamics. Neuron 9:1-13.

Glenn LL, Steriade M (1982) Discharge rate and excitability of cortically projecting intralaminar thalamic neurons during waking and sleep states. J Neurosci 2:1387-1404.

Gray CM (1994) Synchronous oscillations in neuronal systems: mechanisms and function. J Comput Neurosci 1:11-38.

Gray CM, König P, Engel AK, Singer W (1989) Stimulus-specific neuronal oscillations in cat visual cortex exhibit inter-columnar synchronization which reflects global stimulus properties. Nature 338:334-337.

Gurewitsch M, Chatschaturian A (1928) Zur Cytoarchitektonik der Grosshirnrinde der Feliden. Z Anat Entwicklungsgesch 87:100-138.

Gutfreund Y, Yarom Y, Segev I (1995) Subthreshold oscillations and resonant frequency in guinea-pig cortical neurons: physiology and modelling. J Physiol (Lond) 483:621-640.

Hassler R, Muhs-Clement K (1964) Architektonischer Aufbau des sensomotorischen and parietalen Cortex der Katze. J Hirnforsch $6: 377-420$.

Jagadeesh B, Gray CM, Ferster D (1992) Visually evoked oscillations of membrane potential in cells of cat visual cortex. Science 257:552-554.

Jasper HH (1936) Cortical excitatory state and synchronism in the control of bioelectric autonomous rhythms. Cold Spring Harb Symp Quant Biol 4:320-332.

Jasper HH (1958) Recent advances in our understanding of ascending activities of the reticular system. In: Reticular formation of the brain (Jasper HH, Proctor LD, Knighton RS, Noshay WC, Costello RT, eds), pp 319-331. Boston: Little \& Brown.

Jones EG (1975) Possible determinants of the degree of retrograde neuronal labelling with horseradish peroxidase. Brain Res 85:249-253.

Kisvárday ZF, Beaulieu C, Eysel UT (1993) Network of GABAergic large basket cells in visual cortex (area 18): implication for lateral disinhibition. J Comp Neurol 327:398-415.

Krnjevic K, Pumain R, Renaud L (1971) The mechanism of excitation by acetylcholine in the cerebral cortex. J Physiol (Lond) 215:247-268.

Lampl I, Yarom Y (1993) Subthresold oscillations of the membrane potential: a functional synchronizing and timing device. J Neurophysiol $70: 2181-2186$.

Llinás RR (1990) Intrinsic electrical properties of mammalian neurons and CNS function. In: Fidia Research Foundation Neuroscience Award Lcctures, vol 4, pp 175-194. New York: Raven.

Llinás RR, Paré D (1991) Of dreaming and wakefulness. Neuroscience 44:521-535.

Llinás R, Ribary U (1993) Coherent $40-\mathrm{Hz}$ oscillation characterizes dream state in humans. Proc Natl Acad Sci USA 90:2078-2081.

Llinás RR, Grace AA, Yarom Y (1991) In vitro neurons in mammalian cortical layer 4 exhibit intrinsic oscillatory activity in the $10-$ to $50-\mathrm{Hz}$ frequency range. Proc Natl Acad Sci USA 88:897-901.

Llinás R, Ribary U, Joliot M, Wang XJ (1994) Content and context in temporal thalamocortical binding. In: Temporal coding in the brain (Buzsáki G, Llinás R, Singer W, Berthoz A, Christen Y, eds), pp 251 272. Berlin: Springer.

Lopes da Silva FH, Van Rotterdam A, Storm van Leeuwen W, Tielen AM (1970) Dynamic characteristics of visual evoked potentials in the dog. II Beta frequency selectivity in evoked potentials and background activity. Electroencephalogr Clin Neurophysiol 29:260-268.

McCormick DA, Prince DA (1986) Mechanism of action of acetylcholine in the guinea-pig cerebral cortex in vitro. J Physiol (Lond) 375:169-194

Metherate R, Cox CL, Ashe AH (1992) Cellular bases of neocortical activation: modulation of neural oscillations by the nucleus basalis and endogenous acetylcholine. J Neurosci 12:4701-4711.

Moruzzi G, Magoun HW (1949) Brain stem reticular formation and activation of the electroencephalogram. Electroencephalogr Clin Neurophysiol 1:455-473. 
Murthy VN, Fetz EE (1992) Coherent 25- to $35-\mathrm{Hz}$ oscillations in the sensorimotor cortex of awake behaving monkeys. Proc Natl Acad Sci USA 89:5670-5674.

Niedermeyer E (1993) The normal EEG of the waking adult. In: Electroencephalography: basic principles, clinical applications, and rclated fields (Niedermeyer E, Lopes da Silva F, eds), pp 131-152. Baltimore: Williams \& Wilkins.

Nuñez A, Amzica F, Steriade M (1992) Voltage-dependent fast (20-40 $\mathrm{Hz}$ ) oscillations in long-axoned neocortical neurons. Neuroscience 51:7-10.

Nuñez A, Amzica F, Steriade M (1993) Electrophysiology of cat association cortical cells in vivo: intrinsic properties and synaptic responses. J Neurophysiol 70:418-430.

Pantev C, Makeig S, Hoke M, Galambos R, Hampson S, Gallen C (1991) Human auditory evoked gamma-band magnetic fields. Proc Natl Acad Sci USA 88:8996-9000.

Rasmusson DD, Clow K, Szerb JC (1994) Modification of neocortical acetylcholine release and electroencephalogram desynchronization due to brainstem stimulation by drugs applied to basal forebrain. Neuroscience 60:665-677.

Salt TE, Meier CL, Seno N, Krucker T, Herrling PL (1995) Thalamocortical and corticocortical excitatory postsynaptic potentials mediated by excitatory amino acid receptors in the cat motor cortex in vivo. Neuroscience 64:433-442.

Scannell JW, Blakemore C, Young MP (1995) Analysis of connectivity in the cat cerebral cortex. J Neurosci 15:1463-1483.

Schwindt PC, Spain JW, Foehring RC, Stafstrom CE, Chubb MC, Crill WE (1988) Multiple potassium conductances and their functions in neurons from cat sensorimotor cortex in vitro. J Neurophysiol $59: 424-449$.

Singer W (1990a) Search for coherence: a basic principle of cortical self-organization. Concepts Neurosci 1:1-26.

Singer W (1990b) Role of acetylcholine in use-dependent plasticity of the visual cortex. In: Brain cholinergic systems (Steriade M, Biesold D, eds), pp 314-336. Oxford: Oxford UP.

Stafstrom CE, Schwindt PV, Flatman JA, Crill WE (1984) Properties of subthreshold response and action potential recorded in layer $V$ neurons from cat sensorimotor cortex in vitro. J Neurophysiol 52:244-263.

Steriade M (1968) The flash-evoked afterdischarge. Brain Res 9:169-212.

Steriade M (1993) Central core modulation of spontaneous oscillations and sensory transmission in thalamocortical systems. Curr Opin Neurobiol 3:619-625.

Steriade M (1995) Two channels in the cerebello-thalamo-cortical system. J Comp Neurol 354:57-70.

Steriade M, Amzica F (1996) Intracortical and corticothalamic coherency of fast spontaneous oscillations. Proc Natl Acad Sci USA, in press.

Steriade M, Demetrescu M (1960) Unspecific systems of inhibition and facilitation of potentials evoked by intermittent light. J Neurophysiol 23:602-617.

Steriade M, Glenn LL (1982) Neocortical and caudate projections of intralaminar thalamic neurons and their synaptic excitation from midbrain reticular core. J Neurophysiol 48:352-371.

Steriade M, McCarley RW (1990) Brainstem control of wakefulness and sleep. New York: Plenum.
Steriade M, Amzica F, Nuñez A (1993a) Cholinergic and noradrenergic modulation of the slow $(\sim 0.3 \mathrm{~Hz})$ oscillation in neocortical cells. J Neurophysiol 70:1384-1400.

Steriade M, Belekhova M, Apostol V (1968) Reticular potentiation of cortical flash-evoked afterdischarge. Brain Res 11:276-280.

Steriade M, Contreras D, Amzica F (1994) Synchronized Sleep oscillations and their paroxysmal developments. Trends Neurosci 17:199-208.

Steriade M, Curró Dossi R, Contreras D (1993b) Electrophysiological properties of intralaminar thalamocortical cells discharging rhythmic $(\sim 40 \mathrm{~Hz})$ spike-bursts at $\sim 1000 \mathrm{~Hz}$ during waking and rapid eye movement sleep. Neuroscience 56:1-9.

Steriade M, Curró Dossi R, Nuñez A (1991a) Network modulation of a slow intrinsic oscillation of cat thalamocortical neurons implicated in sleep delta waves: cortical potentiation and brainstem cholinergic suppression. J Neurosci 11:3200-3217.

Steriade M, Curró Dossi R, Paré D, Oakson G (1991b) Fast oscillations $(20-40 \mathrm{~Hz})$ in thalamocortical systems and their potentiation by mesopontine cholinergic nuclei in the cat. Proc Natl Acad Sci USA 88:4396-4400.

Steriade M, Datta S, Paré D, Oakson G, Curró Dossi R (1990a) Neuronal activities in brainstem cholinergic nuclei related to activation processes in thalamocortical systems. J Neurosci 10:2541-2559.

Steriade M, Jones EG, Llinás RR (1990b) Thalamic oscillations and signaling. New York: Wiley.

Steriade M, Jones EG, McCormick DA (1996) Thalamus. Oxford: Elsevier, in press.

Steriade M, McCormick DA, Sejnowski TJ (1993c) Thalamocortical oscillations in the sleeping and arousal brain. Science 262:679-685.

Steriade M, Nuñez A, Amzica F (1993d) A novel slow ( $<1 \mathrm{~Hz}$ ) oscillation of neocortical neurons in vivo: depolarizing and hyperpolarizing components. J Neurosci 13:3252-3265.

Steriade M, Nuñez A, Amzica F (1993e) Intracellular analysis of relations between the slow $(<1 \mathrm{~Hz})$ neocortical oscillation and other sleep rhythms of the electroencephalogram. J Neurosci 13:3266-3283.

Stcriadc M, Parent A, Hada J (1984) Thalamic projections of nuclcus reticularis thalami of cat: a study using horseradish peroxidase and double fluorescent tracers. J Comp Neurol 229:531-547.

Thomson AM, Girdlestone D, West DC (1989) A local circuit neocortical synapse that operates via both NMDA and non-NMDA receptors. Br J Pharmacol 96:406-408.

Uchida S, Maloney T, Feinberg I (1994) Sigma (12-16 Hz) and beta $(20-28 \mathrm{~Hz})$ EEG discriminate NREM and REM sleep. Brain Res 659:243-248.

Wang Z, McCormick DA (1993) Control of firing mode of corticotectal and corticopontine layer $\mathrm{V}$ burst-generating neurons by norepinephrinc, acctylcholinc, and 1S, 3R-ACPD. J Ncurosci 13:2199-2216.

Williams JA, Comisarow J, Day J, Fibiger HC, Reiner PB (1994) Statedependent release of acetylcholine in rat thalamus measured by in vivo microdyalisis. J Neurosci 14:5236-5242.

Ylinen A, Bragin A, Nádasdy Z, Jandó G, Szábo I, Sik A, Buzsáki G (1995) Sharp wave-associated high-frequency oscillation $(200 \mathrm{~Hz})$ in the intact hippocampus: network and intracellular mechanisms. J Neurosci $15: 30-46$. 